December 27, 2001

\title{
On Variations in Discrete Mechanics and Field Theory
}

\author{
Han-Ying Guo \\ Institute of Theoretical Physics, Chinese Academia of Sciences, \\ P.O. Box 2735, Beijing 100080, China \\ $\mathrm{Ke} \mathrm{Wu}$ \\ Department of Mathematics, Capital Normal University, \\ Beijing 100037, China \\ Institute of Theoretical Physics, Chinese Academia of Sciences, \\ P.O. Box 2735, Beijing 100080, China
}

\begin{abstract}
Some problems on variations are raised for classical discrete mechanics and field theory and the difference variational approach with variable step-length is proposed motivated by Lee's approach to discrete mechanics and the difference discrete variational principle for difference discrete mechanics and field theory on regular lattice. Based upon Hamilton's principle for the vertical variations and double operation of vertical exterior differential on action, it is shown that for both continuous and variable step-length difference cases there exists the nontrivial Euler-Lagrange cohomology as well as the necessary and sufficient condition for symplectic/multi-symplectic structure preserving properties is the relevant Euler-Lagrange 1-form is closed in both continuous and difference classical mechanics and field theory. While the horizontal variations give rise to the relevant identities or relations of the Euler-Lagrange equation and conservation law of the energy/energy-momentum tensor for continuous or discrete systems. The total variations are also discussed. Especially, for those discrete cases the variable step-length of the difference is determined by the relation between the Euler-Lagrange equation and conservation law of the energy/energy-momentum tensor. In addition, this approach together with difference version of the Euler-Lagrange cohomology can be applied not only to discrete Lagrangian formalism but also to the Hamiltonian formalism for difference mechanics and field theory.
\end{abstract}




\section{INTRODUCTION}

Variation problems play a fundamental, even central, role in various types of continuous mechanics (see, for example, [8], [4], [1]) and field theories for both Lagrangian and Hamiltonian formalisms, which can be transformed each other in many cases via Legendre transformation. A lot of important issues such as the equations of motions, the (intrinsic) symplectic or multisymplectic preserving properties, conservation laws associated with certain symmetries, topological properties etc. are very closely related to the variation problems with fixed or variable domains. On the other hand, however, there are some open questions relevant to variation problems in different kinds of discrete mechanics, field theories as well as corresponding symplectic and multisymplectic algorithms, although discrete variation problems still play an important role, particularly, for the discrete Lagrangian formalism of these discrete systems. In fact, for a long period, there had been no any discrete variation approach available to both discrete Lagrangian and Hamiltonian formalisms that relate each other by discrete Legendre transformation until the difference discrete variational approach has been proposed very recently [12], [14].

As far as discretized version with difference for mechanics is concerned, Lee suggested a discrete variational approach to discrete Lagrangian mechanics and relevant algorithm in early 1980's [19], 20], 21]. In Lee's approach the time steps are variable in view of time being a dynamical variable in order to preserve the energy discretely. Veselov [28], [24] also proposed the discrete variational principle by the end of 1980's, which is almost the same as Lee's approach except without taking variation with respect to the discrete time so that it does not keep the conservation of the energy discretely in general. In addition, both approaches are merely available to discrete Lagrangian mechanics and nothing to do with discrete Hamiltonian mechanics.

On the other hand, Ruth [26] and Feng [10] proposed the symplectic algorithm for Hamiltonian mechanics. In this algorithm (for a review, see [27]), the time step-length is fixed and the symplectic preserving property is discretely kept. However, the discrete version of energy conservation can not be maintained discretely in general. The symplectic algorithm plays very important role in computational mathematics and its applications cover various branches in sciences. It is well known that the progresses of symplectic algorithm promote further development of the structure-preserving algorithms. In these algorithms, the time step-length is always being fixed so that the price paid by keeping structure preserving is the loss of other conservation laws for the continuous cases in general.

Structure preserving criterion: It is widely accepted that the discrete systems should be thought as the discrete counterparts of the corresponding continuous systems. However, in order to discretize continuous systems, a guide line in needed. In Feng's first paper on the symplectic algorithm, he wrote a working hypothesis: "It is natural to look forward to those discrete systems which preserve as much as possible the intrinsic properties of the continuous system." [10] In fact, this statement should be regarded as a criterion, the structure-preserving criterion, for constructing mostly quarried one in all kinds of structurepreserving algorithms. However, in order to carry through this criterion, it is needed to know how to answer the following simple questions.

Problem 1: What are the most important intrinsic "structures" in continuous systems,

such as classical mechanics and field theory, that should also be maintained in the course of discretization? What are the discrete counterparts of these "structures" and how to preserve 
them in certain discrete version? What about the lowest price has to be paid in the course of discretization?

It is well known that there are two classes of conservation laws in canonical conservative mechanics. The first class of conservation laws is that of phase-area conservation laws characterized by the symplectic preserving property and another class is related to energy and all first integrals of the canonical equations. Thus, the following questions can apparently be raised.

Problem 2: Is it possible to establish a kind of discrete mechanics and/or structurepreserving algorithms in such a way that they not only discretely preserve the symplectic property but also some other conservation laws, specially the energy conservation? Do these discrete systems can be established by a discrete variational approach? Does this discrete variational approach can be applied to both discrete Lagrangian and Hamiltonian formalisms?

Although in Lee's approach, it should be able to prove that in addition to discrete energy conservation the symplectic structure is also preserved since Lee's approach is a discrete variational approach Đ. But the framework of either Lee's approach or Veselov's one can not be applied to the discrete Hamiltonian systems. To our knowledge, therefore, these problems are still open, at least partly.

In the cases of discrete field theories and multisymplectic algorithms, the symplectic algorithm has been generalized to the multisymplectic one in what is called "Hamiltonian formalism" [5]. On the other hand, Veselov's discrete variation approach to the discrete mechanics has also been generalized to field theories in Lagrangian formalism to get so-called "variational multisymplectic integrators" [22], [23]. In both approaches, the step-lengths are fixed so that the energy-momentum tensor cannot be conserved in general although the multisymplectic structure preserving property in field theory can be maintained discretely in certain manner. Thus a set of similar questions can also be raised to the discrete field theory and multisymplectic algorithms.

Problem 3: Is it possible to establish a discrete variational approach to describe a kind of discrete field theories and/or multisymplectic algorithms in such a way that not only the multisymplectic property is discretely preserved but also the conservation laws such as energy-momentum conservation law are discretely maintained in certain version? Is it possible to apply such an approach in both discrete Lagrangian and Hamiltonian formalisms? To our knowledge, these problems are still open as well.

As was just mentioned, for the difference discrete variational approach to discrete mechanics and field theory with fixed step-length differences, it can be applied to both Lagrangian and Hamiltonian formalisms [12], [14]. The key point of this approach is to regard the differences with fixed step-length as a kind of entire geometric objects, which play an analogical role with the one played by derivative in continuous cases.

Problem 4: Is it possible to generalize the difference discrete variational approach with fixed step-length differences to the one with varied step-length differences so as to the discrete

\footnotetext{
${ }^{1}$ In [18] this problem has been partly solved by defining a conserved discrete energy in Veselov's approach. The complete resolving to this problem in Lee's framework has been made until very recently in [6], [7].
} 
energy conservation law in certain version may be kept together with the symplectic and/or multisymplectic preserving properties?

In order to carry through the structure-preserving criterion, we present an approach to the discrete variation problems. Namely, the difference variational approach with variable step-lengths named variable difference variational approach to these problems in discrete mechanics and field theory. In other wards, this approach is available to the discrete total variations with keeping the step-length of differences to be varied by an equation from the variation of the discrete action with respect to discrete time and/or discrete space coordinates. As was mentioned, this approach is a natural generalization of the difference variational approach with fixed step-lengths proposed recently in [12], [14 for the ordinary discrete variation problems with fixed discrete domain. In fact, the approach in [12], [14] is just discrete vertical variation so that it is natural to keep the step-length being fixed. The most important key point of this approach is that in the course of calculation of variation problems in discrete mechanics and field theory, the differences with variable step-lengths are kept as entire geometric objects as much as possible. Consequently, this discrete variation approach not only keep the advantage of Lee's discrete variation, which conserves the energy of the system discretely, but also the advantage in variation in Veselov type, which is symplectic or multisymplectic. In addition, this variable difference variation approach can be applied not only to the Lagrangian formalism but the Hamiltonian formalism as well for both discrete mechanics and discrete field theory, since the discrete canonical "momenta" and discrete version of Legendre transformation can be introduced in terms of variable steplength differences. For simplicity, we consider in this paper the discrete Lagrangian of first order of difference only.

The paper is organized as follows. In section 2 , we recall the total variation problems in Lagrangian and Hamiltonian mechanics. In section 3, we present the variable difference variational approach and deal with the difference Lagrangian and Hamiltonian mechanics. In section 4, we recall briefly the total variation problems in Lagrangian and Hamiltonian field theory with generic variables. In section 5, we apply the variable difference discrete variational approach to the total discrete variation problems in difference discrete Lagrangian and Hamiltonian field theory with generic variables. Finally, we end with some remarks.

\section{GENERAL VARIATIONS FOR CLASSICAL MECHANICS}

Let us recall briefly the general or total variation calculus with variable domain in classical mechanics.

Let $t \in T \simeq R$ be the time, $M$ an $n$-dimensional configuration space. Consider a fibre bundle $E(T, Q, \pi)$ with projection $\pi: E \rightarrow T$ on $T, \pi^{-1}: t \rightarrow Q_{t}$ isomorphic to $M$ is the fibre on $t \in T$. Denote $\Gamma(E)$ the sections on $E, T E$ the tangent bundle of $E, T_{v} E \subset T E$ the vertical sub-bundle of $T E$, etc..

\section{A. Variations in Lagrangian mechanics}

We first consider the Lagrangian mechanics. The Lagrangian of the system is denoted as $L\left(q^{i}(t), \dot{q}^{i}(t) ; t\right), i=1, \cdots, n$, which is a mapping from $T E$ to $R$. For simplicity, the Lagrangian is of the first order. The action functional is 


$$
S\left(\left[q^{i}(t)\right] ; t_{1}, t_{2}\right)=\int_{t_{1}}^{t_{2}} d t L\left(q^{i}(t), \dot{q}^{i}(t) ; t\right) .
$$

Here $q^{i}$ 's are coordinates in the fiber, $q^{i}(t)$ describes a curve $C_{a}^{b}$ with ending points $a$ and $b, t_{a}=t_{1}, t_{b}=t_{2}$, along which the motion of the system is assumed to take place, and $\dot{q}^{i}(t)=d q^{i}(t) / d t$.

Let us consider the general variation of $q^{i}(t)$

$$
q^{i}(t) \rightarrow q^{\prime i}\left(t^{\prime}\right)=q^{i}(t)+\delta_{t} q^{i}(t)
$$

accompanied with an infinitesimal re-parameterization of time $t$

$$
t \rightarrow t^{\prime}(t)=t+\delta t .
$$

Here $\delta_{t} q^{i}(t)$ denotes the total variation that can be divided into two parts,

$$
\begin{aligned}
\delta_{t} q^{i}(t) & =\delta_{v} q^{i}(t)+\delta_{h} q^{i}(t), \\
\delta_{v} q^{i}(t) & =q^{\prime i}(t)-q^{i}(t), \\
\delta_{h} q^{i}(t) & =q^{\prime i}\left(t^{\prime}\right)-q^{\prime i}(t) \\
& =q^{i}\left(t^{\prime}\right)-q^{i}(t)+O\left(\delta^{2}\right)=\delta t \frac{d}{d t} q^{i}(t)+O\left(\delta^{2}\right),
\end{aligned}
$$

where $\delta_{v} q^{i}(t)$ denotes the equal time part variation or the vertical one and $\delta_{h} q^{i}(t)$ the horizontal part along the fibre induced by the re-parameterization of the time $t$ (2.3). If we introduce a variational vector field on $T$

$$
\xi(t):=\delta t \frac{d}{d t},
$$

the horizontal variation $\delta_{h} q^{i}(t)$ is the Lie derivative of $q^{i}(t)$ with respect to the variation vector field (2.5).

Similarly,

$$
\begin{aligned}
\delta_{t} \dot{q}^{i}(t) & =\left(\delta_{v}+\delta_{h}\right) \dot{q}^{i}(t), \\
\delta_{v} \dot{q}^{i}(t) & =\dot{q}^{i(}(t)-\dot{q}^{i}(t), \\
\delta_{h} \dot{q}^{i}(t) & =\frac{d}{d t^{\prime}} q^{i(}\left(t^{\prime}\right)-\frac{d}{d t^{\prime}} q^{\prime i}(t) \\
& =\frac{d}{d t^{\prime}} q^{i}\left(t^{\prime}\right)-\frac{d}{d t} q^{i}(t)+O\left(\delta^{2}\right)=\delta t \frac{d}{d t} \dot{q}^{i}(t)+O\left(\delta^{2}\right) .
\end{aligned}
$$

Note that $\delta_{h} \dot{q}^{i}(t)$ is also the Lie derivative of $\dot{q}^{i}(t)$ with respect to the variational vector field (2.5). In fact, this is true for a kind of functionals of $q^{i}(t), \dot{q}^{i}(t)$ and $t$ :

$$
\delta_{h} F\left(q^{i}(t), \dot{q}^{i}(t), t\right)=L_{\xi} F\left(q^{i}(t), \dot{q}^{i}(t), t\right) .
$$

To the time change (2.3) is associated the change in the measure in (2.1) given by the Jacobi formula

$$
d t^{\prime}=\frac{\partial t^{\prime}}{\partial t} d t=\left(1+\frac{d}{d t} \delta t\right) d t
$$

i.e.

$$
\delta(d t)=d(t+\delta t)-d t=d t \frac{d}{d t} \delta t .
$$


It is easy to see that this change in the measure is also the Lie derivative of the measure with respect to the variation vector (2.5):

$$
\delta(d t)=L_{\xi} d t=d L_{\xi} t=d \delta t .
$$

Here the commuting property between $d$ on $T$ and the Lie derivative is used.

Now the Lagrangian is changed as

$$
L\left(q^{i}(t), \frac{d}{d t} q^{i}(t) ; t\right) \rightarrow L\left(q^{\prime i}\left(t^{\prime}\right), \frac{d}{d t^{\prime}} q^{i}\left(t^{\prime}\right) ; t^{\prime}\right)=L\left(q^{i}(t), \frac{d}{d t} q^{i}(t) ; t\right)+\delta_{t} L
$$

and the action is also deformed to

$$
\begin{aligned}
S\left(\left[q^{i}\left(t^{\prime}\right)\right] ; t_{1}^{\prime}, t_{2}^{\prime}\right) & =\int_{t_{1}^{\prime}}^{t_{2}^{\prime}} d t^{\prime} L\left(q^{i}\left(t^{\prime}\right), \frac{d}{d t^{\prime}} q^{\prime i}\left(t^{\prime}\right) ; t^{\prime}\right) \\
& =\int_{t_{1}}^{t_{2}} \frac{\partial t^{\prime}}{\partial t} d t\left\{L\left(q^{i}(t), \dot{q}^{i}(t) ; t\right)+\delta_{t} L\right\} \\
& =\int_{t_{1}}^{t_{2}} d t\left\{L+\left(\frac{d}{d t} \delta t\right) L+\delta_{t} L\right\} \\
& =S\left(\left[q^{i}(t)\right] ; t_{1}, t_{2}\right)+\delta_{t} S
\end{aligned}
$$

A more or less straightforward calculation shows

$$
\delta_{t} S=\int_{t_{1}}^{t_{2}} d t\left\{\left[L_{q^{i}}\right] \delta_{t} q^{i}+\left[\frac{d}{d t} H+\frac{\partial}{\partial t} L\right] \delta t+\frac{d}{d t}\left(\frac{\partial L}{\partial \dot{q}^{j}} \delta_{t} q^{j}-H \delta t\right)\right\}
$$

where $\left[L_{q^{i}}\right]$ is the Euler-Lagrange operator and $H$ is the energy (Hamiltonian)

$$
\left[L_{q^{i}}\right]:=\frac{\partial L}{\partial q^{i}}-\frac{d}{d t}\left(\frac{\partial L}{\partial \dot{q}^{i}}\right), \quad H:=\frac{\partial L}{\partial \dot{q}^{i}} \dot{q}^{i}-L .
$$

The vertical and horizontal variations should be separated as the independent ones and this leads to

$$
\delta_{v} S=\int_{t_{1}}^{t_{2}} d t\left\{\left[L_{q^{i}}\right] \delta_{v} q^{i}+\frac{d}{d t}\left(\frac{\partial L}{\partial \dot{q}^{j}} \delta_{v} q^{j}\right)\right\}
$$

and

$$
\delta_{h} S=\int_{t_{1}}^{t_{2}} d t\left\{\left[L_{q^{i}}\right] \delta_{h} q^{i}+\left[\frac{d}{d t} H+\frac{\partial}{\partial t} L\right] \delta t+\frac{d}{d t}\left(\frac{\partial L}{\partial \dot{q}^{j}} \delta_{h} q^{j}-H \delta t\right)\right\}
$$

For the vertical part, the Hamilton's principle leads to the Euler-Lagrange equation if $\left.\delta_{v} q^{j}\right|_{t_{1}}=\left.\delta_{v} q^{j}\right|_{t_{2}}=0$

$$
\frac{\partial L}{\partial q^{i}}-\frac{d}{d t}\left(\frac{\partial L}{\partial \dot{q}^{i}}\right)=0
$$

For the horizontal part, however, it is easy to check

$$
\begin{aligned}
& {\left[L_{q^{i}}\right] \dot{q}^{i}+\frac{d}{d t} H+\frac{\partial}{\partial t} L=0,} \\
& \vartheta:=\frac{\partial L}{\partial \dot{q}^{j}} \delta_{h} q^{j}-H \delta t=L \delta t .
\end{aligned}
$$

Therefore, 


$$
\delta_{h} S=\int_{t_{1}}^{t_{2}} d t \frac{d}{d t}(L \delta t)=0 .
$$

This is just the invariance of the action $S$ with respect to the re-parameterization of time. Of course, from the first equation of (2.17), it still follows the conservation law for the energy if and only if the Euler-Lagrange equation is satisfied and $L$ does not depend on $t$ manifestly.

If the identities in (2.17) is employed directly, it follows (see, for example, [8]) that

$$
\delta_{t} S=\int_{t_{1}}^{t_{2}} d t\left\{\left[L_{q^{i}}\right] \delta_{v} q^{i}+\frac{d}{d t}\left(\frac{\partial L}{\partial \dot{q}^{j}} \delta_{v} q^{j}+L \delta t\right)\right\}
$$

A quantity now can be defined

$$
J:=\frac{\partial L}{\partial \dot{q}^{j}} \delta_{v} q^{j}+L \delta t
$$

the invariance of $S$ under the re-parameterization of $t$, i.e. $\delta_{t} S=0$, leads to the conservation of the quantity $J$ if and only if the Euler-Lagrange equation is satisfied:

$$
\frac{d}{d t} J=0
$$

\section{Remark 2.1:}

Introducing an exterior differential operator $d_{v}$ along the fibre that satisfies

$$
d_{v}^{2}=0, \quad\left\{d_{v}, d_{h}\right\}=0, \quad d:=d_{v}+d_{h},
$$

where $d_{h}$ and $d$ is the nilpotent exterior differential operator on $T^{*} T$ and $T^{*} E$ respectively as was as a vertical variational vector field

$$
\xi_{q}:=\delta_{v} q^{i}(t) \frac{\partial}{\partial q^{i}},
$$

then

$$
\delta_{v} q^{i}(t)=i_{\xi_{q}} d_{v} q^{i}=i_{\xi_{q}} d q^{i}
$$

By means of the vertical variational vector field (2.23) on $T Q, \delta_{v} S$ can also be expressed as its contraction with 1 -form $d_{v} S \in T^{*} Q$

$$
i_{\xi_{q}} d_{v} S=\delta_{v} S
$$

We may calculate $d_{v} S \in T^{*} Q$. Since $d_{v}$ commutes with the integral of $d t$ (see also, for example, the functional differential calculus in [25]), it is straightforward to get

$$
\left.d_{v} S=\int_{t_{1}}^{t_{2}} d t\left\{\left[L_{q^{i}}\right] d_{v} q^{i}+\frac{d}{d t} \theta\right)\right\}
$$

where $\theta$ is the Lagrange 1 -form

$$
\theta:=\frac{\partial L}{\partial \dot{q}^{i}} d_{v} q^{i} .
$$


Now by contracting with the vertical variational vector field (2.23) it follows straightforwardly $\delta_{v} S$ in (2.14).

Furthermore, due to the nilpotency of $d_{v}$, it is easy to get

$$
d_{v} \mathcal{E}+\frac{d}{d t} \omega=0
$$

where $\mathcal{E}$ is called the Euler-Lagrange 1-form [12, [13], [14, defined by

$$
\mathcal{E}\left(q^{i}(t), \dot{q}^{i}(t) ; t\right):=\left[L_{q^{i}}\right] d_{v} q^{i}
$$

$\omega$ is the symplectic 2-form and in local coordinates:

$$
\omega:=d_{v} \theta=\frac{\partial^{2} L}{\partial q^{j} \partial \dot{q}^{i}} d_{v} q^{j} \wedge d_{v} q^{i}+\frac{\partial^{2} L}{\partial \dot{q}^{j} \partial \dot{q}^{i}} d_{v} \dot{q}^{j} \wedge d_{v} q^{i} .
$$

From (2.29), 2.26) and (2.28), the following theorem can be proved [12], [13], [14]:

Theorem 1: For all Lagrangian of a kind of systems with first order of derivatives on the bundle $E(T, Q, \pi)$

1. The following Euler-Lagrange cohomology is nontrivial:

$$
H_{L M}:=\left\{\mathcal{E} \mid d_{v} \mathcal{E}=0\right\} /\left\{\mathcal{E} \mid \mathcal{E}=d_{v} \alpha\right\}
$$

where $\alpha=\alpha\left(q^{i}(t), \dot{q}^{i}(t) ; t\right)$ is an arbitrary function of $\left(q^{i}(t), \dot{q}^{i}(t) ; t\right)$.

2. The necessary and sufficient condition for conservation of the symplectic 2-form, i.e.

$$
\frac{d}{d t} \omega=0
$$

is the corresponding Euler-Lagrange 1-form being closed.

\section{Remark 2.2:}

From the definition of the Lie derivative it can be seen that the horizontal variations are given by the Lie derivative with respect to the variational vector field.

Let $\xi$ be a vector field on $T, \exp (\lambda \xi)$ be the flow with parameter $\lambda$, i.e. the one-parameter diffeomorphism group, induced by $\xi, f$ a differential or a vector on $T$. The infinitesimal change of $f$ under flow is described by its Lie derivative with respect to the vector field $\xi$

$$
L_{\xi} f(t):=\lim _{\lambda \rightarrow 0} \frac{1}{\lambda}\left\{\phi_{\lambda}^{*} f(\exp (\lambda \xi) t)-f(t)\right\}=\left.\frac{d}{d \lambda}\right|_{\lambda=0}\left(\phi_{\lambda}^{*} f\left(t^{\prime}\right)\right), \quad t^{\prime}=\exp (\lambda \xi) t .
$$

Here $\phi_{\lambda}^{*}$ is the bull-back or the inverse differential for the differential form or vector respectively.

Taking $\xi=\xi(t)=\xi_{t}$ in (2.5), it follows that the Lie derivative of $f(t)$ with respect to $\xi_{t}$ gives rise to the horizontal variation of $f(t)$.

On the other hand, the time variation $\delta t$ can be expressed by the contraction between the variational vector field (2.5) and 1 -form $d_{h} t$ on $T^{*} T$, i.e. $d t$ on $T^{*} E$

$$
i_{\xi_{t}} d_{h} t=\delta t
$$


It is also feasible to express the variation $\delta_{h} q^{i}(t)$ as contraction of a horizontal variation vector field $\xi_{h}$ with $d_{v} q^{i}$ or $d q^{i}$. To this purpose, $\xi_{h, q}$ along the fibre with respect to horizontal variations of $q^{i}(t)$

$$
\xi_{h, q}:=\delta_{h} q^{i}(t) \frac{\partial}{\partial q^{i}}
$$

should be introduced. Combining with the vector field $\xi_{t}$ in (2.5), the general horizontal variational vector field $\xi_{h}$ should be defined as

$$
\xi_{h}:=\xi_{t}+\xi_{h, q}=\delta t \frac{\partial}{\partial t}+\delta_{h} q^{i}(t) \frac{\partial}{\partial q^{i}} .
$$

Its contraction with $d_{v} q^{i}$ or $d q^{i}$ leads to

$$
i_{\xi_{h}} d q^{i}=d q^{i} \cdot \xi_{h}=\delta_{h} q^{i}(t)
$$

In general, for any functional of $q^{i}(t)$ and $\dot{q}^{i}(t), F\left(q^{i}(t), \dot{q}^{i}(t)\right): T Q \rightarrow R$, its (horizontal) variation induced by $(2.3)$ is

$$
\begin{aligned}
F\left(q^{i}(t), \dot{q}^{i}(t)\right) \rightarrow F\left(q^{i}\left(t^{\prime}\right), \frac{d}{d t^{\prime}} q^{i}\left(t^{\prime}\right)\right) & =F\left(q^{i}(t), \dot{q}^{i}(t)\right)+\delta_{h} F\left(q^{i}(t), \dot{q}^{i}(t)\right), \\
\delta_{h} F\left(q^{i}(t), \dot{q}^{i}(t)\right) & =i_{\xi_{h}} d F\left(q^{i}(t), \dot{q}^{i}(t)\right) .
\end{aligned}
$$

\section{Remark 2.3:}

For the total variation, a total variational vector field for $q^{i}(t)$ along the fibre can also be introduced

$$
\begin{aligned}
\xi_{\text {total }}:=\xi_{v}+\xi_{h} & =\delta t \frac{\partial}{\partial t}+\delta_{t} q^{i}(t) \frac{\partial}{\partial q^{i}} \\
& =\delta t \frac{\partial}{\partial t}+\left(\delta_{v} q^{i}(t)+\delta t \frac{d}{d t} q^{i}(t)\right) \frac{\partial}{\partial q^{i}}
\end{aligned}
$$

whose contraction with $d q^{i}$ leads to the total variation $\delta_{t} q^{i}(t)$

$$
i_{\xi_{\text {total }}} d q^{i}=d q^{i} \cdot \xi_{\text {total }}=\delta_{t} q^{i}(t)
$$

If we introduce the Lagrangian 1-from

$$
\mathbf{L}:=L\left(q^{i}, \dot{q}^{i}, t\right) d t
$$

and take $0=d^{2} \mathbf{L}$, it is easy to see that the theorem 1 still holds. This means that the total variations keep the Euler-Lagrange cohomology as well as the necessary and sufficient condition for symplectic structure preserving property in classical mechanics.

\section{Remark 2.4:}

In some literatures (see, for example, [9]), it is required that Hamilton's principle holds for the total variation of the action, i.e. $\delta_{t} S=0$, and regard $\delta_{t} q^{i}$ and $\delta t$ as independent variations. Thus it follows the Euler-Lagrange equation, the conservation relation for the energy and the surface term 


$$
\begin{aligned}
& \frac{\partial L}{\partial q^{i}}-\frac{d}{d t}\left(\frac{\partial L}{\partial \dot{q}^{i}}\right)=0, \\
& \frac{d}{d t} H+\frac{\partial}{\partial t} L=0, \\
& \vartheta=\frac{\partial L}{\partial \dot{q}^{j}} \delta_{t} q^{j}-H \delta t .
\end{aligned}
$$

If $\frac{\partial}{\partial t} L=0$, i.e. the system is conservative, the energy $H$ is conserved. However, $\delta_{t} q^{i}$ is actually dependent on $\delta t$. Therefore, it would be better to regard $\delta_{v} q^{i}$ and $\delta t$ as independent variations.

\section{B. Variations in Hamiltonian mechanics}

The action principle should, of course, be applied to the Hamiltonian mechanics. In order to transfer to the Hamiltonian formalism, we introduce a set of conjugate momenta from the Lagrangian $L\left(q^{i}(t), \dot{q}^{i}(t) ; t\right)$

$$
p_{j}=\frac{\partial L}{\partial \dot{q}^{j}}
$$

and take a Legendre transformation to get the Hamiltonian

$$
H:=H\left(q^{i}, p_{j} ; t\right)=p_{k} \dot{q}^{k}-L\left(q^{i}, \dot{q}^{j} ; t\right) .
$$

Now the action functional can be expressed as

$$
S\left(\left[p_{i}(t)\right],\left[q^{i}(t)\right] ; t_{1}, t_{2}\right)=\int_{t_{1}}^{t_{2}} d t\left\{p_{k} \dot{q}^{k}-H\left(q^{i}, p_{j} ; t\right)\right\} .
$$

The total variation of the action can be calculated

$$
\begin{aligned}
\delta_{t} S & =\delta_{v} S+\delta_{h} S, \\
\delta_{v} S & =\int_{t_{1}}^{t_{2}} d t\left\{\left[H_{p_{i}}\right] \delta_{v} p_{i}-\left[H_{q^{i}}\right] \delta_{v} q^{i}+\frac{d}{d t}\left(p_{i} \delta_{v} q^{i}\right)\right\}, \\
\delta_{h} S & =\int_{t_{1}}^{t_{2}} d t\left\{\left[H_{p_{i}}\right] \delta_{h} p_{i}-\left[H_{q^{i}}\right] \delta_{h} q^{i}+\left[\frac{d}{d t} H-\frac{\partial}{\partial t} H\right] \delta t+\frac{d}{d t}\left(p_{i} \delta_{h} q^{i}-H \delta t\right)\right\},
\end{aligned}
$$

where $\left[H_{p_{i}}\right],\left[H_{q^{i}}\right]$ are canonical operators

$$
\left[H_{p_{i}}\right]:=\dot{q}^{i}-\frac{\partial H}{\partial p_{i}}, \quad\left[H_{q^{i}}\right]:=\dot{p}_{i}+\frac{\partial H}{\partial q^{i}} .
$$

Thus, the stationary requirement for the vertical variation of the action $\delta_{v} S=0$ leads to the canonical equations

$$
\dot{q}^{i}=\frac{\partial H}{\partial p_{i}}, \quad \dot{p}_{i}=-\frac{\partial H}{\partial q^{i}} .
$$

While the time re-parameterization invariance of the action, i.e. $\delta_{h} S=0$ gives rise to an identity on the condition of the energy

$$
\left[H_{p_{i}}\right] \dot{p}_{i}-\left[H_{q^{i}}\right] \dot{q}^{i}+\frac{d}{d t} H-\frac{\partial}{\partial t} H \equiv 0,
$$

and the boundary term that leads to so called "extended symplectic potential" is in fact a total divergence

$$
\int_{t_{1}}^{t_{2}} d t \frac{d}{d t}\left(p_{i} \delta_{h} q^{i}-H \delta t\right)=\int_{t_{1}}^{t_{2}} d t \frac{d}{d t}(L \delta t)=0 .
$$

Similar to the Lagrangian mechanics, all remarks in last subsection can be made for the Hamiltonian formalism. Especially, the theorem 1 can also be established here. 


\section{GENERAL VARIATIONS FOR DISCRETE MECHANICS}

We have proposed a difference variational principle for the (vertical) variation in discrete Lagrangian mechanics in view of the differences with fixed time step-length being regarded as entire variables [12], [14]. One of advantages of this approach is that it is available to both Lagrangian and Hamiltonian formalism for the discrete mechanics. This approach can also be generalized to the total variation for the differences with variable time step-length being regarded as entire variables in such a way that the variable time step-length should be determined by an equation given by the variation problem with variable discrete integral domain. In Lee's wards, discrete time is regarded as a dynamical variable.

Consider the case that "time" $t$ is difference discretized

$$
t \in R \rightarrow t \in T_{D}=\left\{\left(t_{k}, t_{k+1}=t_{k}+\Delta t_{k}, \quad k \in Z\right)\right\}
$$

and the step-lengths $\Delta t_{k}$ are determined by a variational equation, while the $n$-dimensional configuration space $M_{k}$ at each moment $t_{k}, k \in Z$, is still continuous and smooth enough.

Let $N$ be the set of all nodes on $T_{D}$ with index set $\operatorname{Ind}(N)=Z, M=\bigcup_{k \in Z} M_{k}$ the configuration space on $T_{D}$ that is at least pierce wisely smooth enough. At the moment $t_{k}$, $\mathcal{N}_{k}$ be the set of nodes neighboring to $t_{k}$. Let $I_{k}$ the index set of nodes of $\mathcal{N}_{k}$ including $t_{k}$. The coordinates of $M_{k}$ are denoted by $q^{i}\left(t_{k}\right)=q^{i(k)}, i=1, \cdots, n . T\left(M_{k}\right)$ the tangent bundle of $M_{k}$ in the sense that difference at $t_{k}$ is its base, $T^{*}\left(M_{k}\right)$ its dual. Let $\mathcal{M}_{k}=\bigcup_{l \in I_{k}} M_{l}$ be the union of configuration spaces $M_{l}$ at $t_{l}, l \in I_{k}$ on $\mathcal{N}_{k}, T \mathcal{M}_{k}=\bigcup_{l \in I_{k}} T M_{l}$ the union of tangent bundles on $M_{k}, F\left(T M_{k}\right)$ and $F\left(T \mathcal{M}_{k}\right)$ the function spaces on each of them respectively, etc.. In the difference variational approach, we will use these notions.

\section{A. Variable difference Lagrangian mechanics}

Let us consider the system with a discrete Lagrangian $L_{D}{ }^{(k)}$ on $F\left(T\left(M_{k} \times T_{D}\right)\right)$. For simplicity, the Lagrangian is of the first order of differences

$$
L_{D}^{(k)}=L_{D}\left(q^{i(k)}, \Delta_{k} q^{i(k)} ; t_{k}\right),
$$

with the difference $\Delta_{k} q^{i(k)}$ of $q^{i(k)}$ at $t_{k}$ defined by

$$
\Delta_{k} q^{i(k)}:=\frac{q^{i(k+1)}-q^{i(k)}}{t_{k+1}-t_{k}} .
$$

The discrete action of the system is given by

$$
S_{D}=\sum_{k \in Z}\left(t_{k+1}-t_{k}\right) L_{D}{ }^{(k)}\left(q^{i(k)}, \frac{q^{i(k+1)}-q^{i(k)}}{t_{k+1}-t_{k}} ; t_{k}\right) .
$$

The discrete total variations for $q^{i(k)}=q^{i}\left(t_{k}\right)$ should be defined as follows

$$
\begin{aligned}
& \delta_{t} q^{i(k)}:=q^{i}\left(t_{k}^{\prime}\right)-q^{i}\left(t_{k}\right)=\delta_{v} q^{i(k)}+\delta_{h} q^{i(k)}, t_{k}^{\prime}=t_{k}+\delta t_{k}, \\
& \delta_{v} q^{i(k)}:=q^{\prime i}\left(t_{k}\right)-q^{i}\left(t_{k}\right), \delta_{v} t_{k}=0, \\
& \delta_{h} q^{i(k)}:=q^{\prime i}\left(t_{k}^{\prime}\right)-q^{\prime i}\left(t_{k}\right)=q^{i}\left(t_{k}^{\prime}\right)-q^{i}\left(t_{k}\right)+O\left(\delta^{2}\right) .
\end{aligned}
$$


It can be shown that horizontal variation $\delta_{h} q^{i(k)}$ is given by

$$
\delta_{h} q^{i(k)}=\delta t_{k} \Delta_{k} q^{i(k)} .
$$

The discrete total variations for $\Delta_{k} q^{i(k)}$ are defined as

$$
\begin{aligned}
& \delta_{t} \Delta_{k} q^{i(k)}:=\frac{q^{\prime i}\left(t_{k+1}^{\prime}\right)-q^{\prime i}\left(t_{k}^{\prime}\right)}{t_{k+1}^{\prime}-t_{k}^{\prime}}-\frac{q^{i}\left(t_{k+1}\right)-q^{i}\left(t_{k}\right)}{t_{k+1}-t_{k}}=\delta_{v} \Delta_{k} q^{i(k)}+\delta_{h} \Delta_{k} q^{i(k)} \\
& \delta_{v} \Delta_{k} q^{i(k)}:=\frac{q^{\prime i}\left(t_{k+1}\right)-q^{\prime i}\left(t_{k}\right)}{t_{k+1}-t_{k}}-\frac{q^{i}\left(t_{k+1}\right)-q^{i}\left(t_{k}\right)}{t_{k+1}-t_{k}}
\end{aligned}
$$

Due to the definition of the difference with variable time step-length (3.3) and the Leibniz law for it

$$
\Delta_{k}\left(f^{(k)} g^{(k)}\right)=\left(\Delta_{k} f^{(k)}\right) g^{(k)}+E f^{(k)}\left(\Delta_{k} g^{(k)}\right),
$$

where $E$ is the shift operator defined as

$$
E f^{(k)}=f^{(k+1)}, \quad E^{-1} f^{(k)}=f^{(k-1)},
$$

it follows

$$
\begin{aligned}
& \delta_{t} \Delta_{k} q^{i(k)}=\Delta_{k}\left(\delta_{t} q^{i(k)}\right)-\left(\Delta_{k} \delta t_{k}\right) \Delta_{k} q^{i(k)} \\
& \delta_{h} \Delta_{k} q^{i(k)}=\delta t_{k+1} \Delta\left(\Delta_{k} q^{i(k)}\right) .
\end{aligned}
$$

Namely,

$$
\begin{aligned}
& \delta_{v} \Delta_{k} q^{i(k)}=\Delta_{k} \delta_{v} q^{i(k)} \\
& \delta_{h} \Delta_{k} q^{i(k)}=\Delta_{k} \delta_{h} q^{i(k)}-\left(\Delta_{k} \delta t_{k}\right) \Delta_{k} q^{i(k)}
\end{aligned}
$$

Using above properties and

$$
\delta_{t}\left(t_{k+1}-t_{k}\right)=\Delta_{k}\left(\delta_{t} t_{k}\right)\left(t_{k+1}-t_{k}\right)
$$

the total variations of the discrete Lagrangian can be calculated as follows

$$
\begin{aligned}
\delta_{t} L_{D}{ }^{(k)} & =\frac{\partial L_{D}{ }^{(k)}}{\partial q^{i(k)}} \delta_{t} q^{i(k)}+\frac{\partial L_{D}{ }^{(k)}}{\partial \Delta_{k} q^{i(k)}} \delta_{t} \Delta_{k} q^{i(k)}+\frac{\partial L_{D}{ }^{(k)}}{\partial t_{k}} \delta_{t} t_{k} \\
& =\left[L_{q^{i(k)}}\right] \delta_{t} q^{i(k)} \\
& +\Delta_{k}\left(p_{i}{ }^{(k)} \Delta_{k} q^{i(k-1)}\right) \delta t_{k}+\frac{\partial L_{D}{ }^{(k)}}{\partial t_{k}} \delta t_{k} \\
& +\Delta_{k}\left(p_{i}{ }^{(k+1)} \delta_{t} q^{i(k)}-p_{i}{ }^{(k)} \Delta_{k} q^{i(k-1)} \delta t_{k}\right)
\end{aligned}
$$

where $\left[L_{q^{i(k)}}\right]$ is the discrete Euler-Lagrange operator

$$
\left[L_{q^{i(k)}}\right]:=\frac{\partial L_{D}{ }^{(k)}}{\partial q^{i(k)}}-\Delta\left(\frac{\partial L_{D}{ }^{(k-1)}}{\partial \Delta q^{i(k-1)}}\right),
$$

and $p_{i}{ }^{(k)}$ the discrete canonical conjugate momenta

$$
p_{i}^{(k)}:=\frac{\partial L_{D}{ }^{(k-1)}}{\partial \Delta q^{i(k-1)}} .
$$

Thus the total variation of action is given by 


$$
\begin{aligned}
\delta_{t} S_{D} & =\sum_{k}\left(t_{k+1}-t_{k}\right)\left\{\left(\Delta \delta t_{k}\right) L_{D}{ }^{(k)}+\delta_{t} L_{D}{ }^{(k)}\right\} \\
& =\sum_{k}\left(t_{k+1}-t_{k}\right)\left\{\left[L_{q^{i(k)}}\right] \delta_{t} q^{i(k)}+\left(\Delta_{k} H_{D}{ }^{(k-1)}+\frac{\partial L_{D}{ }^{(k)}}{\partial t_{k}}\right) \delta t_{k}\right. \\
& \left.+\Delta_{k}\left(p_{i}{ }^{(k+1)} \delta_{t} q^{i(k)}-H_{D}{ }^{(k-1)} \delta t_{k}\right)\right\}
\end{aligned}
$$

where $H_{D}{ }^{(k)}$ is the difference Hamiltonian that can be introduced through the discrete Legendre transformation

$$
H_{D}^{(k)}:=p_{i}{ }^{(k+1)} \Delta_{t} q^{i(k)}-L_{D}^{(k)} .
$$

Thus the total variation of the discrete action (3.16) can be written as

$$
\begin{aligned}
\delta_{t} S_{D} & =\delta_{v} S_{D}+\delta_{h} S_{D} \\
\delta_{v} S_{D} & =\sum_{k}\left(t_{k+1}-t_{k}\right)\left[L_{q^{i(k)}}\right] \delta_{v} q^{i(k)}+\Delta\left(p_{i}{ }^{(k+1)} \delta_{v} q^{i(k)}\right) \\
\delta_{h} S_{D} & =\sum_{k}\left(t_{k+1}-t_{k}\right)\left\{\left[L_{q^{i(k)}}\right] \delta_{h} q^{i(k)}+\left(\Delta H_{D}{ }^{(k-1)}+\frac{\partial L_{D}{ }^{(k)}}{\partial t_{k}}\right) \delta t_{k}\right. \\
& \left.+\Delta\left(p_{i}{ }^{(k+1)} \delta_{h} q^{i(k)}-H_{D}{ }^{(k-1)} \delta t_{k}\right)\right\} .
\end{aligned}
$$

The variational principle requires $\delta_{v} S_{D}=0$ and the discretized re-parameterization invariance with respect to discrete time may also leads to $\delta_{h} S_{D}=0$ if this invariance does exist. Thus it follows the discrete Euler-Lagrange equations for $q^{i(k)}$ 's

$$
\frac{\partial L_{D}^{(k)}}{\partial q^{i(k)}}-\Delta\left(\frac{\partial L_{D}^{(k-1)}}{\partial \Delta q^{i(k-1)}}\right)=0,
$$

and the equation for the variable time step-length

$$
\left(\frac{\partial L_{D}{ }^{(k)}}{\partial q^{i(k)}}-\Delta\left(\frac{\partial L_{D}^{(k-1)}}{\partial \Delta q^{i(k-1)}}\right)\right) \Delta q^{i(k)}+\Delta H_{D}{ }^{(k-1)}-\frac{\partial H_{D}{ }^{(k)}}{\partial t_{k}}=0 .
$$

It is more or less straightforward to show that if the time step-length is fixed the equation (3.20) has no solution in general even if the Lagrangian does not depend on discrete time manifestly. In other wards, for the conservative discrete Lagrangian mechanics the time steplength should be variable in general so as to the energy of the system can be kept conserved discretely.

\section{Remark 3.1:}

We may introduce exterior differential operators $\hat{d}, d_{v}$ and $\hat{d}_{h}$ on $T^{*}\left(M \times T_{D}\right), T^{*} M$ and $T^{*} T_{D}$ respectively. They are nilpotent and satisfy

$$
\hat{d}=d_{v}+\hat{d}_{h}, \quad\left\{d_{v}, \hat{d}_{h}\right\}=0 .
$$

Especially, $\hat{d}_{h}$ is due to the difference on $T_{D}$ and satisfy Leibniz's law for ordinary forms 2 .

\section{Remark 3.2:}

\footnotetext{
${ }^{2}$ It is needed some noncommutative differential calculus to completely clarify the properties of $\hat{d}_{h}$. For the case that $\Delta t$ is fixed, the noncommutative differential calculus can be found in [15], [16]. For the case of variable time steps, similar noncommutative differential calculus can be established [17.
} 
Actually, analog to the case with fixed time steps [12], [14], it can be established the difference version for the Euler-Lagrange cohomology and the necessary and sufficient condition for the difference conservation law of the discrete symplectic 2-form.

From $\delta_{v} S_{D}$ in (3.18), it is easy to see that we may take $d_{v}$ on $S_{D}$ to get

$$
d_{v} S_{D}=\sum_{k}\left(t_{k+1}-t_{k}\right) d_{v} L_{D}{ }^{(k)}, \quad d_{v} L_{D}{ }^{(k)}=\mathcal{E}_{D}{ }^{(k)}+\Delta_{k} \theta_{D}{ }^{(k)},
$$

where $\mathcal{E}_{D}{ }^{(k)}, \theta_{D}{ }^{(k)}$ are the discrete Euler-Lagrange 1-form and symplectic potential 1-form respectively

$$
\mathcal{E}_{D}{ }^{(k)}:=\left[L_{q^{i(k)}}\right] d_{v} q^{i(k)}, \quad \theta_{D}{ }^{(k)}:=p_{i}{ }^{(k+1)} d_{v} q^{i(k)}
$$

Then due to the nilpotency of $d_{v}$, it is straightforward to get

$$
d_{v} \mathcal{E}_{D}{ }^{(k)}+\Delta_{k} \omega_{D}{ }^{(k)}=0, \quad \omega_{D}{ }^{(k)}:=d_{v} \theta_{D}{ }^{(k)}=d_{v} p_{i}{ }^{(k+1)} \wedge d_{v} q^{i(k)} .
$$

Therefore, we may get the discrete version for the theorem 1 [12], [13], [14]:

Theorem 2: For all discrete Lagrangian of a kind of systems with first order differences on the bundle $E\left(T_{D}, Q, \pi\right) \simeq M \times T_{D}$,

1. The following discrete version of the Euler-Lagrange cohomology is nontrivial: $H_{D C M}:=\{$ Closed Euler-Lagrange forms $\} /\{$ Exact Euler-Lagrange forms $\}$.

2. The necessary and sufficient condition for conservation of the discrete symplectic 2-form, i.e.

$$
\Delta_{k} \omega_{D}{ }^{(k)}=0
$$

is the corresponding discrete Euler-Lagrange 1-form being closed.

\section{Remark 3.3:}

In this paper, $T_{D}$ is an infinite chain. It is reasonable to consider an interval on $T_{D}$. We will publish the issues on this topic elsewhere.

\section{B. Variable difference Hamiltonian mechanics}

Now we consider the total difference variation on the phase space in the discrete Hamiltonian formalism with variable (time step-length) difference.

In order to transfer to the discrete Hamiltonian formalism, it is needed to introduce the discrete canonical conjugate momenta according to the equation (3.15) and express the discrete Lagrangian by the discrete Hamiltonian via Legentre transformation (3.17). Thus, the discrete action can be expressed as

$$
\begin{aligned}
S_{D} & =\sum_{k}\left(t_{k+1}-t_{k}\right) L_{D}{ }^{(k)}\left(q^{i(k)}, \frac{q^{i(k+1)}-q^{i(k)}}{t_{k+1}-t_{k}}, t_{k}\right) \\
& =\sum_{k}\left(t_{k+1}-t_{k}\right)\left(p_{i}{ }^{(k+1)} \Delta_{t} q^{i(k)}-H_{D}{ }^{(k)}\right) .
\end{aligned}
$$

And its total variation reads 


$$
\begin{aligned}
\delta_{t} S_{D}= & \delta_{v} S_{D}+\delta_{h} S_{D} \\
= & \sum_{k}\left(t_{k+1}-t_{k}\right)\left\{\left(\Delta q^{i(k)}-\frac{\partial \mathcal{H}_{D}{ }^{(k)}}{\partial p_{i}{ }^{(k+1)}}\right) \delta_{t} p_{i}{ }^{(k+1)}\right. \\
& -\left(\Delta p_{i}{ }^{(k)}+\frac{\partial H_{D}{ }^{(k)}}{\partial q^{i(k)}}\right) \delta_{t} q^{i(k)}+\left(\Delta H_{D}{ }^{(k-1)}+\frac{\partial L_{D}{ }^{(k)}}{\partial t_{k}}\right) \delta t_{k} \\
& \left.+\Delta\left(p_{i}{ }^{(k)} \delta_{t} q^{i(k)}-H_{D}{ }^{(k-1)} \delta t_{k}\right)\right\} .
\end{aligned}
$$

Similar to the discrete Lagrangian formalism, Hamilton's principle requires $\delta_{v} S_{D}=0$ and the discretized re-parameterization invariance with respect to discrete time may also lead to $\delta_{h} S_{D}=0$ if this invariance does exist. Thus it follows the discrete canonical equations for $p_{i}{ }^{(k)}$ 's and $q^{i(k)}$ 's

$$
\Delta q^{i(k)}=\frac{\partial H_{D}{ }^{(k)}}{\partial p_{i}{ }^{(k+1)}}, \quad \Delta p_{i}{ }^{(k)}=-\frac{\partial H_{D}{ }^{(k)}}{\partial q^{i(k)}},
$$

the equation for the variable time step-length

$$
\left(\Delta q^{i(k)}-\frac{\partial H_{D}{ }^{(k)}}{\partial p_{i}{ }^{(k+1)}}\right) \Delta p_{i}{ }^{(k+1)}-\left(\Delta p_{i}{ }^{(k)}+\frac{\partial H_{D}{ }^{(k)}}{\partial q^{i(k)}}\right) \Delta q^{i(k)}+\Delta H_{D}{ }^{(k-1)}-\frac{\partial H_{D}(k)}{\partial t_{k}}=0 .
$$

It is also more or less straightforward to show that if the time step-length is fixed the equation (3.29) has no solution in general even if the Hamiltonian does not depend on discrete time manifestly. In other wards, for the conservative discrete Hamiltonian mechanics the time step-length should be variable so as to the energy of the system can be kept conserved discretely. In [19], 20], [21], [11], [18], this issue has been studied.

It should be mentioned that all remarks in last subsection can be made here and the theorem 2 can also be established for the discrete Hamiltonian formalism.

\section{GENERAL VARIATIONS FOR FIELD THEORY}

Consider a bundle $E(X, Q, \pi)$, the fibre $Q \simeq M$. For simplicity, let $X=X^{(1, n-1)}$ be an $n$ dimensional Minkowskian space as base manifold with coordinates $x^{\mu},(\mu=0, \cdots, n-1), M$ the configuration space on $X^{(1, n-1)}$ with a set of generic (scalar) fields $u^{\alpha}(x),(\alpha=1, \cdots, r)$, $T M$ the tangent bundle of $M$ with coordinates $\left(u^{\alpha}, u_{\mu}^{\alpha}\right)$, where $u_{\mu}^{\alpha}=\frac{\partial u^{\alpha}}{\partial x^{\mu}}, F(T M)$ the function space on $T M$ etc.

We also assume these fields to be free of constraints. In fact, the approach here can easily be applied to other cases.

\section{A. General variation in Lagrangian formalism}

The Lagrangian of the theory is supposed to be the first order of derivatives of the fields and dependent to the coordinates manifestly, i.e. $L\left(u^{\alpha}, u_{\mu}^{\alpha} ; x^{\mu}\right)$, and the action is

$$
S\left(\left[u^{\alpha}(x)\right] ; x^{\mu}\right)=\int_{\Omega} d^{4} x L\left(u^{\alpha}, u_{\mu}^{\alpha} ; x^{\mu}\right) .
$$

Let us consider the variations of the fields, i.e. total variation $\delta_{t} u^{\alpha}$, vertical one $\delta_{v} u^{\alpha}$ and horizontal one $\delta_{h} u^{\alpha}$ : 


$$
\begin{aligned}
& u^{\alpha} \rightarrow u^{\prime \alpha}\left(x^{\prime}\right)=u^{\alpha}(x)+\delta_{t} u^{\alpha}(x), \\
& \delta_{t} u^{\alpha}=\delta_{v} u^{\alpha}+\delta_{h} u^{\alpha} \text {, } \\
& \delta_{v} u^{\alpha}(x):=u^{\alpha}(x)-u^{\alpha}(x) \text {, } \\
& \delta_{h} u^{\alpha}(x):=u^{\prime \alpha}\left(x^{\prime}\right)-u^{\prime \alpha}(x)=u^{\alpha}\left(x^{\prime}\right)-u^{\alpha}(x)+O\left(\delta^{2}\right) \\
& =\delta x^{\mu} \partial_{\mu} u^{\alpha}(x)
\end{aligned}
$$

accompanying with the coordinates' infinitesimal continuous transformation

$$
x^{\mu} \rightarrow x^{\prime \mu}=x^{\mu}+\delta x^{\mu} .
$$

The corresponding changes in the derivative of fields $u_{\mu}^{\alpha}$ are

$$
\begin{gathered}
\frac{\partial}{\partial x^{\mu}} u^{\alpha}(x) \rightarrow \frac{\partial}{\partial x^{\prime \mu}} u^{\prime \alpha}\left(x^{\prime}\right)=\frac{\partial}{\partial x^{\mu}} u^{\alpha}(x)+\delta_{t}\left(\frac{\partial}{\partial x^{\mu}} u^{\alpha}(x)\right), \\
\delta_{t}\left(\frac{\partial}{\partial x^{\mu}} u^{\alpha}(x)\right)=\delta_{v}\left(\frac{\partial}{\partial x^{\mu}} u^{\alpha}(x)\right)+\delta_{h}\left(\frac{\partial}{\partial x^{\mu}} u^{\alpha}(x)\right), \\
\delta_{v}\left(\frac{\partial}{\partial x^{\mu}} u^{\alpha}(x)\right):=\frac{\partial}{\partial x^{\mu}} u^{\prime \alpha}(x)-\frac{\partial}{\partial x^{\mu}} u^{\alpha}(x), \\
\delta_{h}\left(\frac{\partial}{\partial x^{\mu}} u^{\alpha}(x)\right)=\delta x^{\nu} \frac{\partial}{\partial x^{\nu}}\left(\frac{\partial}{\partial x^{\mu}} u^{\alpha}(x)\right) .
\end{gathered}
$$

Now the action (4.1) is also changed as follows

$$
\begin{aligned}
S\left(\left[u^{\alpha}(x)\right] ; x^{\mu}\right) \rightarrow S^{\prime} & \left(\left[u^{\prime \alpha}\left(x^{\prime}\right)\right] ; x^{\prime \mu}\right)=\int_{\Omega^{\prime}} d^{n} x^{\prime} L^{\prime}\left(u^{\prime \alpha}\left(x^{\prime}\right), u_{\mu^{\prime}}^{\prime \alpha}\left(x^{\prime}\right) ; x^{\prime \mu}\right) \\
& =\int_{\Omega} d^{n} x \operatorname{det}\left(\frac{\partial x^{\prime}}{\partial x}\right)\left\{L\left(u^{\alpha}(x), u_{\mu}^{\alpha}(x) ; x^{\mu}\right)+\delta_{t} L\right\} \\
& =S\left(\left[u^{\alpha}(x)\right] ; x^{\mu}\right)+\delta_{t} S .
\end{aligned}
$$

Using Jacobi formula for the measure

$$
d^{n} x^{\prime}=\operatorname{det}\left(\frac{\partial x^{\prime}}{\partial x}\right) d^{n} x=\left(1+\frac{\partial \delta x^{\mu}}{\partial x^{\mu}}\right) d^{n} x
$$

we get

$$
\begin{aligned}
\delta_{t} S\left(\left[u^{\alpha}(x)\right] ; x^{\mu}\right)= & \int_{\Omega} d^{n} x\left\{\partial_{\mu} \delta x^{\mu} L+\delta_{t} L\right\} \\
= & \int_{\Omega} d^{n} x\left\{\left[L_{u^{\alpha}}\right] \delta_{t} u^{\alpha}+\left(\partial^{\mu} T_{\mu \nu}+\frac{\partial L}{\partial x^{\nu}}\right) \delta x^{\nu}\right. \\
& \left.+\partial_{\mu}\left(\frac{\partial L}{\partial\left(\partial_{\mu} u^{\alpha}\right)} \delta_{t} u^{\alpha}-T^{\mu}{ }_{\nu} \delta x^{\nu}\right)\right\}, \\
\delta_{v} S\left(\left[u^{\alpha}(x)\right] ; x^{\mu}\right)= & \int_{\Omega} d^{n} x\left\{\left[L_{u^{\alpha}}\right] \delta_{v} u^{\alpha}+\partial_{\mu}\left(\frac{\partial L}{\partial\left(\partial_{\mu} u^{\alpha}\right)} \delta_{v} u^{\alpha}\right)\right\}, \\
\delta_{h} S\left(\left[u^{\alpha}(x)\right] ; x^{\mu}\right)= & \int_{\Omega} d^{n} x\left\{\left[L_{u^{\alpha}}\right] \delta_{h} u^{\alpha}+\left(\partial^{\mu} T_{\mu \nu}+\frac{\partial L}{\partial x^{\nu}}\right) \delta x^{\nu}\right. \\
& \left.+\partial_{\mu}\left(\frac{\partial L}{\partial\left(\partial_{\mu} u^{\alpha}\right)} \delta_{h} u^{\alpha}-T^{\mu}{ }_{\nu} \delta x^{\nu}\right)\right\} \\
= & \int_{\Omega} d^{n} x \partial_{\mu}\left(L \delta x^{\mu}\right),
\end{aligned}
$$

where $\left[L_{u^{\alpha}}\right], T_{\mu \nu}$ are the Euler-Lagrange operator and energy-momentum tensor respectively

$$
\begin{aligned}
{\left[L_{u^{\alpha}}\right] } & :=\frac{\partial L}{\partial u^{\alpha}}-\partial_{\mu}\left(\frac{\partial L}{\partial u_{\mu}^{\alpha}}\right) \\
T_{\mu \nu} & :=\frac{\partial L}{\partial\left(\partial^{\mu} u^{\alpha}\right)} \partial_{\nu} u^{\alpha}-L \eta_{\mu \nu} .
\end{aligned}
$$

Thus $\delta_{t} S=0$, i.e. $\delta_{v} S=0$ due to Hamilton's principle and $\delta_{h} S=0$ due to the invariance of re-parameterization of the coordinates that suppose to keep the metric [3, requires to regard $\delta_{v} u^{\alpha}$ and $\delta x^{\mu}$ as independent components and gives rise to the Euler-Lagrange equation

\footnotetext{
${ }^{3}$ Thus the symmetry is the Poincarè transformations. In general, the general coordinate transformations can be considered and all formulae become covariant.
} 


$$
\frac{\partial L}{\partial u^{\alpha}}-\partial_{\mu}\left(\frac{\partial L}{\partial u_{\mu}^{\alpha}}\right)=0
$$

as well as an identity between the Euler-Lagrange operator and conservation property for the energy-momentum tensor

$$
\left(\frac{\partial L}{\partial u^{\alpha}}-\partial_{\mu}\left(\frac{\partial L}{\partial u_{\mu}^{\alpha}}\right)\right) \partial_{\nu} u^{\alpha}+\partial^{\mu} T_{\mu \nu}+\partial_{\nu} L=0 .
$$

This equation shows that the energy-momentum tensor is conserved if and only if the EulerLagrange equation is satisfied and the Lagrangian does not depend manifestly on the coordinates.

The boundary term is as follows

$$
\int_{\Omega} d^{n} x \frac{\partial}{\partial x^{\mu}}\left(\left(\frac{\partial L}{\partial u_{\mu}^{\alpha}}\right) \delta_{v} u^{\alpha}+L \delta x^{\mu}\right)=\int_{\partial \Omega}\left(\left(\frac{\partial L}{\partial u_{\mu}^{\alpha}}\right) \delta_{v} u^{\alpha}+L \delta x^{\mu}\right) d \sigma^{\mu}=0 .
$$

If we define a current

$$
J^{\mu}:=\left(\frac{\partial L}{\partial u_{\mu}^{\alpha}}\right) \delta_{v} u^{\alpha}+L \delta x^{\mu},
$$

the equation (4.13) leads to an continuity equation for the current if and only if the EulerLagrange equation is satisfied:

$$
\frac{\partial}{\partial x^{\mu}} J^{\mu}=0
$$

In fact, this current is just the Noether current with respect to the invariance of the action under re-parameterization of the right-angle coordinates on $M$.

\section{Remark 4.1:}

Introducing an exterior differential operator $d_{v}$ along the fibre $Q \simeq M$ that satisfies

$$
d_{v}{ }^{2}=0, \quad\left\{d_{v}, d_{h}\right\}=0, \quad d:=d_{v}+d_{h},
$$

where $d_{h}$ and $d$ is the nilpotent exterior differential operator on $T^{*} X$ and $T^{*} E, E=M \times X$ respectively as was as a vertical variational vector field

$$
\xi_{u}:=\delta_{v} u^{\alpha}(x) \frac{\partial}{\partial u^{\alpha}}
$$

then

$$
\delta_{v} u^{\alpha}(x)=i_{\xi_{u}} d_{v} u^{\alpha}=i_{\xi_{u}} d u^{\alpha} .
$$

By means of the vertical variational vector field (4.17) on $T Q, \delta_{v} S$ can also be expressed as its contraction with 1 -form $d_{v} S \in T^{*} Q$

$$
i_{\xi_{u}} d_{v} S=\delta_{v} S
$$

We may calculate $d_{v} S \in T^{*} Q$. Since $d_{v}$ commutes with the integral and $d^{n} x$ (see also, for example, the functional differential calculus in [25]), it is straightforward to get 


$$
\left.d_{v} S=\int_{\Omega} d^{n} x\left\{\left[L_{u^{\alpha}}\right] d_{v} u^{\alpha}+\frac{\partial}{\partial x^{\mu}} \theta^{\mu}\right)\right\}
$$

where $\theta^{\mu}$ are the Lagrange 1-forms

$$
\theta^{\mu}:=\frac{\partial L}{\partial u_{\mu}^{\alpha}} d_{v} u^{\alpha}
$$

Now by contracting with the vertical variational vector field (4.17) it follows straightforwardly $\delta_{v} S$.

Furthermore, due to the nilpotency of $d_{v}$, it is easy to get

$$
d_{v} \mathcal{E}_{u}+\frac{\partial}{\partial x^{\mu}} \omega^{\mu}=0
$$

where $\mathcal{E}_{u}$ is the Euler-Lagrange 1 -form defined by

$$
\mathcal{E}_{u}\left(u^{\alpha}(x), u_{\mu}^{\alpha}(x) ; x\right):=\left[L_{u^{\alpha}}\right] d_{v} u^{\alpha},
$$

$\omega^{\mu}$ are the multi-symplectic 2 -forms and in local coordinates:

$$
\omega^{\mu}:=d_{v} \theta^{\mu}=\frac{\partial^{2} L}{\partial u^{\alpha} \partial u_{\mu}^{\beta}} d u^{\alpha} \wedge d u^{\beta}+\frac{\partial^{2} L}{\partial u_{\nu}^{\alpha} \partial u_{\mu}^{\beta}} d u_{\nu}^{\alpha} \wedge d u^{\beta} .
$$

From the definition (4.23), equations $(4.20)$ and (4.22), the following theorem [12], [13], |14 holds:

Theorem 3: For all Lagrangian of a kind of field theories with first order of derivatives on the bundle $E(X, Q, \pi)$,

1. The following Euler-Lagrange cohomology is nontrivial:

$$
H_{C F T}:=\left\{\mathcal{E}_{u} \mid d \mathcal{E}_{u}=0\right\} /\left\{\mathcal{E}_{u} \mid \mathcal{E}_{u}=d \beta\right\}
$$

where $\beta=\beta\left(u^{\alpha}(x), u_{\mu}^{\alpha}(x) ; x\right)$ is an arbitrary function of $\left(u^{\alpha}(x), u_{\mu}^{\alpha}(x) ; x\right)$.

2. The necessary and sufficient condition for conservation of the multi-symplectic 2forms, i.e.

$$
\frac{\partial}{\partial x^{\mu}} \omega^{\mu}=0
$$

is the corresponding Euler-Lagrange 1-form being closed.

\section{Remark 4.2:}

From the definition of the Lie derivative it can be seen that the horizontal variations are given by the Lie derivative with respect to the variational vector field.

Let $\xi$ be a vector field on $X, \exp (\lambda \xi)$ be the flow with parameter $\lambda$, i.e. the one-parameter diffeomorphism group, induced by $\xi, f$ a differential or a vector on $X$. The infinitesimal change of $f$ under flow is described by its Lie derivative with respect to the vector field $\xi$

$$
L_{\xi} f(x):=\lim _{\lambda \rightarrow 0} \frac{1}{\lambda}\left\{\phi_{\lambda}^{*} f(\exp (\lambda \xi) x)-f(x)\right\}=\left.\frac{d}{d \lambda}\right|_{\lambda=0}\left(\phi_{\lambda}^{*} f\left(x^{\prime}\right)\right), \quad x^{\prime}=\exp (\lambda \xi) x .
$$


Here $\phi_{\lambda}^{*}$ is the bull-back or the inverse differential for the differential form or vector respectively.

Taking horizontal variational vector field for the coordinates $\xi=\xi(x)=\xi_{x}$

$$
\xi(x):=\delta x^{\mu} \frac{\partial}{\partial x^{\mu}}
$$

it follows that the Lie derivative of $f(x)$ with respect to $\xi_{x}$ gives rise to the horizontal variation of $f(x)$.

On the other hand, the coordinate variations $\delta x^{\mu}$ can be expressed by the contraction between the variational vector field (4.27) and 1-form $d_{h} x^{\mu}$ on $T^{*} X$, i.e. $d x^{\mu}$ on $T^{*} E$

$$
i_{\xi_{x}} d_{h} x^{\mu}=\delta x^{\mu}
$$

It is also feasible to express the variation $\delta_{h} u^{\alpha}(x)$ as contraction of a horizontal variation vector field $\xi_{h}$ with $d_{v} u^{\alpha}$ or $d u^{\alpha}$. To this purpose, $\xi_{h, u}$ along the fibre with respect to horizontal variations of $u^{\alpha}(x)$

$$
\xi_{h, u}:=\delta_{h} u^{\alpha}(x) \frac{\partial}{\partial u^{\alpha}}
$$

should be introduced. Combining with the vector field $\xi_{x}$ in (4.27), the general horizontal variational vector field $\xi_{h}$ should be defined as

$$
\xi_{h}:=\xi_{x}+\xi_{h, u}=\delta x^{\mu} \frac{\partial}{\partial x^{\mu}}+\delta_{h} u^{\alpha}(x) \frac{\partial}{\partial u^{\alpha}} .
$$

Its contraction with $d_{v} u^{\alpha}$ or $d u^{\alpha}$ leads to

$$
i_{\xi_{h}} d u^{\alpha}=d u^{\alpha} \cdot \xi_{h}=\delta_{h} u^{\alpha}(t) .
$$

In general, for any functional of $u^{\alpha}(x)$ and $u_{\mu}^{\alpha}(x), F\left(u^{\alpha}(x), u_{\mu}^{\alpha}(x)\right): T Q \rightarrow R$, its (horizontal) variation induced by (4.3) is

$$
\begin{aligned}
F\left(u^{\alpha}(x), u^{\alpha}(x)\right) \rightarrow F\left(u^{\alpha}\left(x^{\prime}\right), \frac{\partial}{\partial x^{\prime \mu}} u^{\alpha}\left(x^{\prime}\right)\right) & =F\left(u^{\alpha}(x), u_{\mu}^{\alpha}(x)\right)+\delta_{h} F\left(u^{\alpha}(x), u_{\mu}^{\alpha}(x)\right), \\
\delta_{h} F\left(u^{\alpha}(x), u_{\mu}^{\alpha}(x)\right) & =i_{\xi_{h}} d F\left(u^{\alpha}(x), u_{\mu}^{\alpha}(x)\right) .
\end{aligned}
$$

\section{Remark 4.3:}

For the total variation, a total variational vector field for $u^{\alpha}(x)$ along the fibre can also be introduced

$$
\begin{aligned}
\xi_{\text {total }}:=\xi_{v}+\xi_{h} & =\delta x^{\mu} \frac{\partial}{\partial x^{\mu}}+\delta_{t} u^{\alpha}(x) \frac{\partial}{\partial u^{\alpha}} \\
& =\delta x^{\mu} \frac{\partial}{\partial x^{\mu}}+\left(\delta_{v} u^{\alpha}(x)+\delta x^{\mu} \frac{\partial}{\partial x^{\mu}} u^{\alpha}(x)\right) \frac{\partial}{\partial u^{\alpha}}
\end{aligned}
$$

whose contraction with $d u^{\alpha}$ leads to the total variation $\delta_{t} u^{\alpha}(x)$

$$
i_{\xi_{\text {total }}} d u^{\alpha}=d u^{\alpha} \cdot \xi_{\text {total }}=\delta_{t} u^{\alpha}(x)
$$




\section{B. General variation in Hamiltonian formalism}

In order to transfer to the Hamiltonian formalism for classical field theory, we first define a set of "momenta" canonically conjugate to the field variables

$$
\pi_{\alpha}(x)=\frac{\partial L}{\partial \dot{u}^{\alpha}}
$$

and take a Legendre transformation to get the Hamiltonian density

$$
H\left(u^{\alpha}, \pi_{\alpha}, \nabla_{a} u^{\alpha}\right)=\pi_{\alpha}(x) \dot{u}^{\alpha}(x)-L\left(u^{\alpha}, \dot{u}^{\alpha}, \nabla_{a} u^{\alpha}\right),
$$

where $\nabla_{a}=\frac{\partial}{\partial x^{a}}, a=1, \cdots, n-1$. The Hamiltonian then is given by

$$
H(t)=\int_{\Sigma} d^{n-1} x H(x)
$$

with the Legendre transformation

$$
H(t)=\int_{\Sigma} d^{n-1} x\left\{\pi_{\alpha}(x) \dot{u}^{\alpha}(x)-L(t)\right\}, \quad L(t)=\int_{\Sigma} d^{n-1} x L,
$$

where $\Sigma \subset \Omega$ is a 3-dimensional simultaneous space-like hypersurface in $\Omega$.

The action $S\left(\left[u^{\alpha}(x)\right] ; x^{\mu}\right)$ (4.1) becomes

$$
S\left(\left[u^{\alpha}(x)\right] ; x^{\mu}\right)=\int_{\Omega} d^{n} x\left\{\pi_{\alpha}(x) \dot{u}^{\alpha}(x)-H\left(u^{\alpha}, \dot{u}^{\alpha}, \nabla_{a} u^{\alpha}\right)\right\} .
$$

The total variation of the action can be calculated similar to that in the last subsection, but here $\pi_{\alpha}(x), u^{\alpha}(x)$ and their derivatives should be varied independently. Then we get

$$
\begin{aligned}
\delta_{t} S\left(\left[u^{\alpha}(x)\right] ; x^{\mu}\right)= & \int_{\Omega} d^{n} x\left\{\partial_{\mu} \delta x^{\mu} L+\delta_{t} L\right\} \\
= & \int_{\Omega} d^{n} x\left\{\left[H_{\pi^{\alpha}}\right] \delta_{t} \pi^{\alpha}-\left[H_{u^{\alpha}}\right] \delta_{t} u^{\alpha}\right. \\
& \left.+\left(\partial^{\mu} T_{\mu \nu}+\frac{\partial H}{\partial x^{\nu}}\right) \delta x^{\nu}-\partial_{\mu}\left(\frac{\partial H}{\partial\left(\partial_{\mu} u^{\alpha}\right)} \delta_{t} u^{\alpha}-T^{\mu}{ }_{\nu} \delta x^{\nu}\right)\right\},
\end{aligned}
$$

where $\left[H_{u^{\alpha}}\right],\left[H_{\pi^{\alpha}}\right]$ are the canonical operators

$$
\begin{aligned}
& {\left[H_{\pi_{\alpha}}\right]:=\dot{u}^{\alpha}(x)-\frac{\partial H}{\partial \pi_{\alpha}},} \\
& {\left[H_{u^{\alpha}}\right]:=\dot{\pi}_{\alpha}(x)+\frac{\partial H}{\partial u^{\alpha}}-\nabla_{a}\left(\frac{\partial H}{\partial\left(\nabla_{a} u^{\alpha}\right)}\right) .}
\end{aligned}
$$

Similar to the case of Lagrangian formalism, $\delta_{t} S=0$, i.e. $\delta_{v} S=0$ and $\delta_{h} S=0$, requires to regard $\delta_{v} u^{\alpha}, \delta_{v} \pi_{\alpha}$ and $\delta x^{\mu}$ as independent components and leads to the canonical field equations

$$
\begin{aligned}
& \dot{u}^{\alpha}(x)=\frac{\partial H}{\partial \pi_{\alpha}}, \\
& \dot{\pi}_{\alpha}(x)=-\frac{\partial H}{\partial u^{\alpha}}+\nabla_{a}\left(\frac{\partial H}{\partial\left(\nabla_{a} u^{\alpha}\right)}\right),
\end{aligned}
$$

and an identity between the canonical operators and conservation property for the energymomentum tensor

$$
\left[H_{\pi^{\alpha}}\right] \partial_{\nu} \pi^{\alpha}-\left[H_{u^{\alpha}}\right] \partial_{\nu} u^{\alpha}+\partial^{\mu} T_{\mu \nu}-\partial_{\nu} H=0 .
$$

It should be mentioned that all remarks in the last subsection can be made here and the theorem 3 can be established as well. 


\section{GENERAL VARIATIONS FOR DISCRETE FIELD THEORY}

We now study the variation problems for the difference discrete field theory with variable step-lengths. For simplicity, we consider the cases of $1+1$ or 2 dimensional flat base manifold, i.e. $X^{1,1}$ or $X^{2}$ endowed with suitable signature of the metrics.

Let $L^{2}$ be a right-angle lattice on $X^{1,1}$ or $X^{2}$ with nodes $x_{\mu}^{(i, j)}=\left(x_{1}^{i}, x_{2}^{j}\right), \mu=1,2,(i, j) \in$ $Z \times Z$ and variable step-lengths on 2-directions $x_{\mu}$ to be determined by discrete variation problems, $N$ be all nodes on $L^{2}$. For a given node with coordinates $x_{\mu}^{(i, j)}, M_{D}:=M^{(i, j)}$ be the piece of configuration space with a set of generic field variables $u^{\alpha}\left(x_{\mu}^{(i, j)}\right)=u^{\alpha(i, j)} \in M_{D}$ at the node $x_{\mu}^{(i, j)}, T M^{(i, j)}$ the tangent bundle of $M^{(i, j)}$ with the set of field variables and their differences $\left(u^{\alpha(i, j)}, \Delta_{\mu} u^{\alpha(i, j)}\right) \in T\left(M^{(i, j)}\right), F\left(T M^{(i, j)}\right)$ the function space on $T M^{(i, j)}$. Let $\mathcal{N}^{(i, j)}$ be the set of nodes neighboring to $x_{\mu}^{(i, j)}$ with index set $I^{(i, j)}=\operatorname{Ind}\left(\mathcal{N}^{(i, j)}\right)$, a set of nodes related to $x_{\mu}^{(i, j)}$ by the differences, $\mathcal{M}^{(i, j)}=\bigcup_{\operatorname{Ind}(N) \mid I^{(i, j)}} M^{(i, j)}$ the union of the pieces of configuration space on $X^{(i, j)}$. $F\left(T\left(\mathcal{M}^{(i, j)}\right)\right)$ function space on $T\left(\mathcal{M}^{(i, j)}\right)$.

Since $L^{2}$ is a right-angle lattice, it should have only two possibilities for the variable steplengths: either equal step-length variation along two direction simultaneously while along each direction the step-lengths are variable, or along one direction the step-length is fixed while along the other it is variable.

\section{A. Variable difference Lagrangian field theory}

The difference Lagrangian for a set of the generic fields $u^{\alpha}, \alpha=1, \cdots, r$, is a functional on $F\left(T\left(M^{(i, j)}\right)\right)$ and suppose to be the first order of differences of the fields for simplicity

$$
L_{D}^{(i, j)}=L_{D}\left(u^{\alpha(i, j)}, \Delta_{\mu} u^{\alpha(i, j)}, x_{\mu}^{(i, j)}\right), \quad \mu=1,2,
$$

where as just mentioned, $x_{\mu}^{(i, j)}=\left(x_{1}^{(i)}, x_{2}^{(j)}\right), u^{\alpha(i, j)}=u^{\alpha}\left(x_{\mu}^{(i, j)}\right)$ and

$$
\Delta_{1} u^{\alpha(i, j)}=\frac{u^{\alpha(i+1, j)}-u^{\alpha(i, j)}}{x_{1}^{(i+1)}-x_{1}^{(i)}}, \quad \Delta_{2} u^{\alpha(i, j)}=\frac{u^{\alpha(i, j+1)}-u^{\alpha(i, j)}}{x_{2}^{(j+1)}-x_{2}^{(j)}} .
$$

The discrete action $S_{D}$ now reads

$$
S_{D}=\sum_{i, j} \Delta_{1} x_{1}^{(i)} \Delta_{2} x_{2}^{(j)} L_{D}^{(i, j)},
$$

where $\Delta_{1} x_{1}^{(i)}=x_{1}^{(i+1)}-x_{1}^{(i)}, \Delta_{2} x_{2}^{(j)}=x_{2}^{(j+1)}-x_{2}^{(j)}$.

Let us consider the coordinates of nodes on the lattice are subject to infinitesimal deformations that still keep $L^{2}$ as a right-angle lattice

$$
x_{\mu}^{(i, j)} \rightarrow x_{\mu}^{\prime}\left(x_{1}^{(i, j)}, x_{2}^{(i, j)}\right)=x_{\mu}^{(i, j)}+\delta x_{\mu}^{(i, j)},
$$

the corresponding changes in the fields are

$$
\begin{aligned}
u^{\alpha}(x)^{(i, j)} \rightarrow & u^{\prime \alpha}\left(x^{\prime}\right)^{(i, j)}=u^{\alpha}(x)^{(i, j)}+\delta_{t} u^{\alpha}(x)^{(i, j)} \\
& \delta_{t} u^{\alpha}(x)^{(i, j)}=\delta_{v} u^{\alpha}(x)^{(i, j)}+\delta_{h} u^{\alpha}(x)^{(i, j)} \\
& \delta_{v} u^{\alpha}(x)^{(i, j)}:=u^{\prime \alpha}(x)^{(i, j)}-u^{\alpha}(x)^{(i, j)} \\
& \delta_{h} u^{\alpha}(x)^{(i, j)}:=u^{\prime \alpha}\left(x^{\prime}\right)^{(i, j)}-u^{\prime \alpha}(x)^{(i, j)} \\
& =u^{\alpha}\left(x^{\prime}\right)^{(i, j)}-u^{\alpha}(x)^{(i, j)}+O\left(\delta^{2}\right) \\
& =\delta x^{\mu(i, j)} \Delta_{\mu} u^{\alpha}(x)^{(i, j)} .
\end{aligned}
$$


For the differences of fields, $\Delta_{\mu} u^{\alpha}(x)^{(i, j)}$, the corresponding changes

$$
\begin{aligned}
\Delta_{\mu} u^{\alpha}(x)^{(i, j)} \rightarrow & \Delta_{\mu}^{\prime} u^{\prime \alpha}\left(x^{\prime}\left(x^{(i, j)}\right)\right)=\Delta_{\mu} u^{\alpha}(x)^{(i, j)}+\delta_{t} \Delta_{\mu} u^{\alpha}(x)^{(i, j)} \\
& \delta_{t} \Delta_{\mu} u^{\alpha}(x)^{(i, j)}=\delta_{v} \Delta_{\mu} u^{\alpha}(x)^{(i, j)}+\delta_{h} \Delta_{\mu} u^{\alpha}(x)^{(i, j)} \\
& \delta_{v} \Delta_{\mu} u^{\alpha}(x)^{(i, j)}:=\Delta_{\mu} u^{\prime \alpha}(x)^{(i, j)}-\Delta_{\mu} u^{\alpha}(x)^{(i, j)} \\
& \delta_{h} \Delta_{\mu} u^{\alpha}(x)^{(i, j)}:=\Delta_{\mu}^{\prime} u^{\prime \alpha}\left(x^{\prime}\right)^{(i, j)}-\Delta_{\mu} u^{\prime \alpha}(x)^{(i, j)} \\
& =\Delta_{\mu} u^{\alpha}\left(x^{\prime}\right)^{(i, j)}-\Delta_{\mu} u^{\alpha}(x)^{(i, j)}+o\left(\delta^{2}\right),
\end{aligned}
$$

can be calculated to get

$$
\begin{aligned}
& \delta_{v} \Delta_{\mu} u^{\alpha}(x)^{(i, j)}=\Delta_{\mu}\left(\delta_{v} u^{\alpha}(x)^{(i, j)}\right) \\
& \delta_{t} \Delta_{\mu} u^{\alpha}(x)^{(i, j)}=\Delta_{\mu} \delta_{t} u^{\alpha}(x)^{(i, j)}-\Delta_{\mu} \delta x^{\nu(i, j)} \cdot \Delta_{\nu} u^{\alpha}(x)^{(i, j)}
\end{aligned}
$$

Using the Leibniz law (3.8) for differences in each direction, it follows

$$
\begin{aligned}
& \delta_{t} \Delta_{1} u^{\alpha}(x)^{(i, j)}=\Delta_{1} \delta_{v} u^{\alpha}(x)^{(i, j)}+\delta x^{\nu(i+1, j)} \cdot \Delta_{1} \Delta_{\nu} u^{\alpha}(x)^{(i, j)} \\
& \delta_{t} \Delta_{2} u^{\alpha}(x)^{(i, j)}=\Delta_{2} \delta_{v} u^{\alpha}(x)^{(i, j)}+\delta x^{\nu(i, j+1)} \cdot \Delta_{2} \Delta_{\nu} u^{\alpha}(x)^{(i, j)}
\end{aligned}
$$

The total variation of discrete action (5.3) can be calculated

$$
\delta_{t} S_{D}=\sum_{i, j} \Delta_{1} x_{1}^{(i)} \cdot \Delta_{2} x_{2}^{(j)}\left(\Delta_{1} \delta x_{1}^{i} L_{D}{ }^{(i, j)}+\Delta_{2} \delta x_{2}^{j} L_{D}{ }^{(i, j)}+\delta_{t} L_{D}{ }^{(i, j)}\right) .
$$

Using formulae

$$
\begin{aligned}
& \Delta_{1} \delta x_{1}^{i} L_{D}{ }^{(i, j)}=\Delta_{1}\left(\delta x_{1}^{i} L_{D}{ }^{(i-1, j)}\right)-\delta x_{1}^{i} \Delta_{1} \mathcal{L}_{D}{ }^{(i-1, j)} \\
& \Delta_{2} \delta x_{2}^{j} L_{D}{ }^{(i, j)}=\Delta_{2}\left(\delta x_{2}^{j} L_{D}{ }^{(i, j-1)}\right)-\delta x_{2}^{j} \Delta_{2} \mathcal{L}_{D}{ }^{(i, j-1)}
\end{aligned}
$$

and

$$
\begin{aligned}
\delta_{t} L_{D}{ }^{(i, j)} & =\frac{\partial \mathcal{L}_{D}{ }^{(i, j)}}{\partial u^{\alpha(i, j)}} \delta_{t} u^{\alpha(i, j)}+\frac{\partial L_{D}{ }^{(i, j)}}{\partial\left(\Delta_{\mu} u^{\alpha(i, j)}\right)} \delta_{t} \Delta_{\mu} u^{\alpha(i, j)}+\frac{\partial L_{D}{ }^{(i, j)}}{\partial x^{\mu(i, j)}} \delta x^{\mu(i, j)} \\
& =\frac{\partial L_{D}{ }^{(i, j)}}{\partial u^{\alpha(i, j)}} \delta_{t} u^{\alpha(i, j)}+\frac{\partial L_{D}{ }^{(i, j)}}{\partial\left(\Delta_{1} u^{\alpha(i, j)}\right)}\left(\Delta_{1} \delta_{t} u^{\alpha(i, j)}-\left(\Delta_{1} \delta x^{\mu(i, j)}\right) \cdot \Delta_{\mu} u^{\alpha(i, j)}\right) \\
& +\frac{\partial L_{D}(i, j)}{\partial\left(\Delta_{2} u^{\alpha(i, j)}\right)}\left(\Delta_{2} \delta_{t} u^{\alpha(i, j)}-\left(\Delta_{2} \delta x^{\mu(i, j)}\right) \cdot \Delta_{\mu} u^{\alpha(i, j)}\right)+\frac{\partial L_{D}(i, j)}{\partial x^{\mu(i, j)}} \delta x^{\mu(i, j)} \\
& =\left[L_{\left.u^{\alpha(i, j)}\right] \delta_{t} u^{\alpha(i, j)}}\right. \\
& +\Delta_{1}\left(\frac{\partial L_{D}(i-1, j)}{\partial\left(\Delta_{1} u^{\alpha(i-1, j)}\right)} \delta_{t} u^{\alpha(i, j)}-\frac{\partial L_{D}{ }^{(i-1, j)}}{\partial\left(\Delta_{1} u^{\alpha(i-1, j)}\right)} \Delta_{\mu} u^{\alpha(i-1, j)} \delta x^{\mu(i, j)}\right) \\
& +\Delta_{2}\left(\frac{\partial L_{D}(i, j-1)}{\partial\left(\Delta_{2} u^{\alpha(i, j-1)}\right)} \delta_{t} u^{\alpha(i, j)}-\frac{\partial L_{D}{ }^{(i, j-1)}}{\partial\left(\Delta_{2} u^{\alpha(i, j-1)}\right)} \Delta_{\mu} u^{\alpha(i, j-1)} \delta x^{\mu(i, j)}\right) \\
& +\Delta_{1}\left(\frac{\partial L_{D}(i-1, j)}{\partial\left(\Delta_{1} u^{\alpha(i-1, j)}\right)} \Delta_{\mu} u^{\alpha(i-1, j)}\right) \delta x^{\mu(i, j)} \\
& +\Delta_{2}\left(\frac{\partial L_{D}(i, j-1)}{\partial\left(\Delta_{2} u^{\alpha(i, j-1)}\right)} \Delta_{\mu} u^{\alpha(i, j-1)}\right) \delta x^{\mu(i, j)} \\
& +\frac{\partial L_{D}{ }^{(i, j)}}{\partial x^{\mu(i, j)}} \delta x^{\mu(i, j)}
\end{aligned}
$$

we get 


$$
\begin{aligned}
& \delta_{t} S_{D}=\delta_{v} S_{D}+\delta_{h} S_{D} \\
& =\sum_{i, j} \Delta_{1} x_{1}^{(i)} \cdot \Delta_{2} x_{2}^{(j)}\left\{\left[L_{u^{\alpha(i, j)}}\right] \delta_{t} u^{\alpha(i, j)}\right. \\
& +\Delta_{1}\left(\frac{\partial L_{D}{ }^{(i-1, j)}}{\partial\left(\Delta_{1} u^{\alpha(i-1, j)}\right)} \delta_{t} u^{\alpha(i, j)}-\left(\frac{\partial L_{D}{ }^{(i-1, j)}}{\partial\left(\Delta_{1} u^{\alpha(i-1, j)}\right)} \Delta_{\mu} u^{\alpha(i-1, j)}-\delta_{1 \mu} L_{D}{ }^{(i-1, j)}\right) \delta x^{\mu(i, j)}\right) \\
& +\Delta_{2}\left(\frac{\partial L_{D}{ }^{(i, j-1)}}{\partial\left(\Delta_{2} u^{\alpha(i, j-1)}\right)} \delta_{t} u^{\alpha(i, j)}-\left(\frac{\partial L_{D}{ }^{(i, j-1)}}{\partial\left(\Delta_{2} u^{\alpha(i, j-1)}\right)} \Delta_{\mu} u^{\alpha(i, j-1)}-\delta_{2 \mu} L_{D}^{(i, j-1)}\right) \delta x^{\mu(i, j)}\right) \\
& +\Delta_{1}\left(\frac{\partial L_{D}(i-1, j)}{\partial\left(\Delta_{1} u^{\alpha(i-1, j)}\right)} \Delta_{\mu} u^{\alpha(i-1, j)}-\delta_{1 \mu} L_{D}{ }^{(i-1, j)}\right) \delta x^{\mu(i, j)} \\
& +\Delta_{2}\left(\frac{\partial L_{D}{ }^{(i, j-1)}}{\partial\left(\Delta_{2} u^{\alpha(i, j-1)}\right)} \Delta_{\mu} u^{\alpha(i, j-1)}-\delta_{2 \mu} L_{D}{ }^{(i, j-1)}\right) \delta x^{\mu(i, j)} \\
& \left.+\frac{\partial L_{D}^{(i, j)}}{\partial x^{\mu(i, j)}} \delta x^{\mu(i, j)}\right\} \\
& =\sum_{i, j} \Delta_{1} x_{1}^{(i)} \cdot \Delta_{2} x_{2}^{(j)}\left\{\left[L_{u^{\alpha(i, j)}}\right] \delta_{t} u^{\alpha(i, j)}\right. \\
& +\Sigma_{\mu, \nu=1,2} \Delta_{\mu}\left(\frac{\partial L_{D}{ }^{(i, j)}}{\partial\left(\Delta_{\mu} u^{(i, j)}\right)} \delta_{t} u^{(i, j)}-E_{\mu}^{-1} T_{D}^{\mu(i, j)} \delta x^{\nu(i, j)}\right) \\
& \left.+\Sigma_{\nu, \mu=1,2}\left(\Delta_{\nu} E_{\nu}^{-1} T_{D_{\mu}}^{\nu(i, j)}+\frac{\partial L_{D}^{(i, j)}}{\partial x^{\mu(i, j)}}\right) \delta x^{\mu(i, j)}\right\},
\end{aligned}
$$

where $E_{\mu}, \mu=1,2,\left[L_{u^{\alpha(i, j)}}\right]$ and $T_{D \mu \nu}{ }^{(i, j)}$ are shift operators, discrete Euler-Lagrange operator and energy-momentum tensor respectively

$$
\begin{gathered}
E_{1} f^{(i, j)}=f^{(i+1, j)}, \quad E_{1}^{-1} f^{(i, j)}=f^{(i-1, j)} \\
E_{2} f^{(i, j)}=f^{(i, j+1)}, \quad E_{2}^{-1} f^{(i, j)}=f^{(i, j-1)} \\
{\left[L_{u^{\alpha(i, j)}}\right]:=\frac{\partial L_{D}{ }^{(i, j)}}{\partial u^{\alpha(i, j)}}-\Delta_{1}\left(\frac{\partial L_{D}{ }^{(i-1, j)}}{\partial\left(\Delta_{1} u^{\alpha(i-1, j)}\right)}\right)-\Delta_{2}\left(\frac{\partial L_{D}{ }^{(i, j-1)}}{\partial\left(\Delta_{2} u^{\alpha(i, j-1)}\right)}\right)} \\
T_{D \mu \nu}{ }^{(i, j)}:=\frac{\partial L_{D}{ }^{(i, j)}}{\partial\left(\Delta^{\mu} u^{\alpha(i, j)}\right)} \Delta_{\nu} u^{\alpha(i, j)}-L_{D}{ }^{(i, j)} \eta_{\mu \nu} .
\end{gathered}
$$

Regarding $\delta_{v} u^{\alpha(i, j)}$ and $\delta x^{\nu(i, j)}$ are independent variational bases, $\delta_{t} S_{D}=0$, or $\delta_{v} S_{D}=0$ and $\delta_{h} S_{D}=0$, lead to the discrete Euler-Lagrange equation

$$
\frac{\partial L_{D}^{(i, j)}}{\partial u^{\alpha(i, j)}}-\Delta_{1}\left(\frac{\partial L_{D}^{(i-1, j)}}{\partial\left(\Delta_{1} u^{\alpha(i-1, j)}\right)}\right)-\Delta_{2}\left(\frac{\partial L_{D}^{(i, j-1)}}{\partial\left(\Delta_{2} u^{\alpha(i, j-1)}\right)}\right)=0
$$

a relation between the Euler-Lagrange operator and the (difference) divergence of the discrete energy-momentum tensor that may determine the step-lengths

$$
\left[L_{u^{\alpha(i, j)}}\right] \Delta_{\nu} u^{\alpha}(x)^{(i, j)}+\Sigma_{\mu=1,2}\left\{\Delta_{\mu} E_{\mu}^{-1} T_{D_{\nu}^{\mu(i, j)}}+\frac{\partial L_{D}^{(i, j)}}{\partial x^{\nu(i, j)}}\right\}=0 .
$$

It is obvious that all these discrete equation, relation and properties have correct continuous limits respectively. Furthermore, due to the discrete Lagrangian (5.1) depends on the differences explicitly, it is possible to introduce the discrete canonical momentum and discrete Legendre transformation to transfer to the discrete Hamiltonian formalism as will be shown in next subsection.

\section{Remark 5.1:}

We may introduce exterior differential operators $\hat{d}, d_{v}$ and $\hat{d}_{h}$ on $T^{*}\left(M \times X_{D}\right), T^{*} M$ and $T^{*} X_{D}$ respectively. They are nilpotent and satisfy 


$$
\hat{d}=d_{v}+\hat{d}_{h}, \quad\left\{d_{v}, \hat{d}_{h}\right\}=0 .
$$

Especially, $\hat{d}_{h}$ is due to the difference on $X_{D}$ and satisfy Leibniz's law for ordinary forms t.

\section{Remark 5.2:}

Actually, analog to the case with fixed step-lengths [12], [14], it can be established the difference version for the Euler-Lagrange cohomology and the necessary and sufficient condition for the difference conservation law of the discrete multi-symplectic 2-forms.

From $\delta_{v} S_{D}$ in (5.12), it is easy to see that we may take $d_{v}$ on $S_{D}$ to get

$$
d_{v} S_{D}=\sum_{(i, j)} \Delta_{1} x_{1}^{i} \Delta_{2} x_{2}^{j} d_{v} L_{D}{ }^{(i, j)}, \quad d_{v} L_{D}{ }^{(i, j)}=\mathcal{E}_{D}{ }^{(i, j)}+\Delta_{\mu} \theta_{D}{ }^{\mu(i, j)}
$$

where $\mathcal{E}_{D}{ }^{(i, j)}, \theta_{D}{ }^{\mu(i, j)}, \mu=1,2$ are the discrete Euler-Lagrange 1-form and multi-symplectic potential 1-forms respectively

$$
\begin{gathered}
\mathcal{E}_{D}^{(i, j)}:=\left[L_{D u^{\alpha(i, j)}}\right] d_{v} u^{\alpha(i, j)} \\
\theta_{D}^{1(i, j)}:=\frac{\partial L_{D}^{(i-1, j)}}{\partial\left(\Delta_{1} u^{\alpha(k-1, l)}\right)} d u^{\alpha(k, l)}, \quad \theta_{D L}{ }^{2(i, j)}:=\frac{\partial L_{D}^{(i, j-1)}}{\partial\left(\Delta_{2} u^{\alpha(k, l-1)}\right)} d u^{\alpha(k, l)}
\end{gathered}
$$

Then due to the nilpotency of $d_{v}$, it is straightforward to get

$$
d_{v} \mathcal{E}_{D}{ }^{(i, j)}+\Delta_{\mu} \omega_{D}{ }^{\mu(i, j)}=0, \quad \omega_{D}{ }^{\mu(i, j)}:=d_{v} \theta_{D}{ }^{\mu(i, j)} .
$$

Therefore, we may get the discrete version for the theorem 3 [12], 14]:

Theorem 4: For all discrete Lagrangian of a kind of discrete field theories with first order of differences on the bundle $E\left(X_{D}, Q, \pi\right) \simeq M \times X_{D}$,

1. The following discrete version of the Euler-Lagrange cohomology is nontrivial:

$H_{D F T}:=\{$ Closed Euler-Lagrange forms $\} /\{$ Exact Euler-Lagrange forms $\}$.

2. The necessary and sufficient condition for conservation of the discrete multi-symplectic 2-forms, i.e.

$$
\Delta_{\mu} \omega_{D}^{\mu(i, j)}=0
$$

is the corresponding discrete Euler-Lagrange 1-form being closed.

\section{Remark 5.3:}

In this paper, $L^{2}$ is an infinite lattice. It is reasonable to consider a finite lattice. We will publish the issues on this topic elsewhere.

\footnotetext{
${ }^{4}$ It is needed some noncommutative differential calculus to completely clarify the properties of $\hat{d}_{h}$. For the case that $\Delta x^{\mu}$ are fixed, this noncommutative differential calculus can be found in [15], [16]. For the case of variable step-lengths, similar noncommutative differential calculus can be established [17].
} 


\section{B. Variable difference Hamiltonian field theory}

Consider $X^{(1,1)}$, on which there is a right-angle lattice $L^{2}$ with variable step-lengths in each direction, is the base space.

We first define a set of the discrete canonical conjugate momenta on the tangent space $T\left(\mathcal{M}^{(i, j)}\right)$ of $\mathcal{M}^{(i, j)}=\bigcup_{\operatorname{Ind}(N) \mid I^{(i, j)}} M^{(i, j)}$ the union of the pieces of configuration space on $X^{(i, j)}$, which are the set of nodes neighboring to the node $x_{\mu}^{(i, j)}$ :

$$
\pi_{\alpha}^{(i, j)}=\frac{\partial L_{D}{ }^{(i-1, j)}}{\partial\left(\Delta_{t} u^{\alpha(i-1, j)}\right)} .
$$

The difference Hamiltonian is introduced through the discrete Legendre transformation

$$
H_{D}{ }^{(i, j)}\left(u^{\alpha(i, j)}, \pi_{\alpha}^{(i+1, j)} ; x^{(i, j)}\right)=\pi_{\alpha}^{(i+1, j)} \Delta_{t} u^{\alpha(i, j)}-L_{D}{ }^{(i, j)} .
$$

The action functional (5.3) now is expressed as

$$
S_{D}=\sum_{(i, j) \in Z \times Z} \Delta_{\mu} x^{\mu(i, j)}\left(\pi_{\alpha}{ }^{(i+1, j)} \Delta_{t} u^{\alpha(i, j)}-H_{D}{ }^{(i, j)}\right) .
$$

The total variation of the action $\delta_{t} S_{D}$ can be calculated and separated into two parts, i.e. the vertical variation $\delta_{v} S_{D}$ and the horizontal variation $\delta_{h} S_{D}$

$$
\begin{gathered}
\delta_{t} S_{D}=\delta_{v} S_{D}+\delta_{h} S_{D}, \\
\delta_{v} S_{D}=\sum_{(i, j) \in Z \times Z} \Delta_{\mu} x^{\mu(i, j)}\left\{\delta_{v} \pi_{\alpha}^{(i+1, j)}\left[H_{\pi_{\alpha}(i+1, j)}\right]-\left[H_{u^{\alpha(i, j)}}\right] \delta_{v} u^{\alpha(i, j)}\right. \\
\left.-\Sigma_{\mu, \nu=1,2} \Delta_{\mu}\left(\frac{\partial H_{D}{ }^{(i, j)}}{\partial\left(\Delta_{\mu} u^{(i, j)}\right)} \delta_{v} u^{(i, j)}\right)\right\}, \\
\delta_{h} S_{D}=\sum_{(i, j) \in Z \times Z} \Delta_{\mu} x^{\mu(i, j)}\left\{\delta_{h} \pi_{\alpha}^{(i+1, j)}\left[H_{\pi_{\alpha}^{(i+1, j)}}\right]-\left[H_{u^{\alpha(i, j)}}\right] \delta_{h} u^{\alpha(i, j)}\right. \\
-\Sigma_{\mu, \nu=1,2} \Delta_{\mu}\left(\frac{\partial H_{D}{ }^{(i, j)}}{\partial\left(\Delta_{\mu} u^{(i, j)}\right)} \delta_{h} u^{(i, j)}+E_{\mu}^{-1} T_{D}^{\mu(i, j)} \delta x^{\nu(i, j)}\right) \\
\left.+\Sigma_{\nu, \mu=1,2}\left(\Delta_{\nu} E_{\nu}{ }^{-1} T_{D}{ }^{\nu}{ }^{(i, j)}-\frac{\partial H_{D}{ }^{(i, j)}}{\partial x^{\mu(i, j)}}\right) \delta x^{\mu(i, j)}\right\} .
\end{gathered}
$$

Here

$$
\begin{aligned}
{\left[H_{\pi_{\alpha}(i+1, j)}\right] } & :=\Delta_{t} u^{\alpha(i, j)}-\frac{\partial H_{D}{ }^{(i, j)}}{\partial \pi_{\alpha}^{(i+1, j)}} \\
{\left[H_{u^{\alpha(i, j)}}\right] } & :=\Delta_{t} \pi_{\alpha}{ }^{(i, j)}+\frac{\partial H_{D}^{(i, j)}}{\partial u^{\alpha(i, j)}}-\Delta_{x}\left(\frac{\partial H_{D}^{(i, j-1)}}{\partial\left(\Delta_{x} u^{\alpha(i, j-1)}\right)}\right) .
\end{aligned}
$$

Regarding $\delta_{v} u^{\alpha(i, j)}, \delta_{v} \pi_{\alpha}{ }^{(i+1, j)}$ and $\delta x^{\nu(i, j)}$ are independent variational bases, $\delta_{v} S_{D}=0$ due to discrete Hamilton's principle and $\delta_{h} S_{D}=0$ due to discreteized re-parameterization invariance on $L^{2}$, i.e. $\delta_{t} S_{D}=0$, lead to the discrete canonical field equations

$$
\begin{aligned}
\Delta_{t} u^{\alpha(i, j)} & =\frac{\partial H_{D}{ }^{(i, j)}}{\partial \pi_{\alpha}(i+1, j)}, \\
\Delta_{t} \pi_{\alpha}{ }^{(i, j)} & =-\frac{\partial H_{D}^{(i, j)}}{\partial u^{\alpha(i, j)}}+\Delta_{x}\left(\frac{\partial H_{D}^{(i, j-1)}}{\partial\left(\Delta_{x} u^{\alpha(i, j-1)}\right)}\right),
\end{aligned}
$$

the canonical form of the relation (5.17) that may determine the step-lengths

$$
\left[H_{\pi_{\alpha}(i+1, j)}\right] \Delta_{\nu} \pi_{\alpha}{ }^{(i+1, j)}-\left[H_{u^{\alpha(i, j)}}\right] \Delta_{\nu} u^{\alpha(i, j)}+\Sigma_{\mu=1,2}\left\{\Delta_{\mu} E_{\mu}^{-1} T_{D}{ }^{\mu(i, j)}-\frac{\partial H_{D}{ }^{(i, j)}}{\partial x^{\nu(i, j)}}\right\}=0 .
$$

It should also be mentioned that all remarks in the last subsection can be made hare and the theorem 4 can also be established in this discrete Hamiltonian formalism for field theory. 


\section{CONCLUDING REMARKS}

In this paper, the variable difference variational approach with variable step-lengths has been proposed. It is a generalized version of the difference variational approach with fixed step-lengths proposed in [12], [14]. The approach has been applied to both Lagrangian and Hamiltonian formalism for discrete mechanics and field theory. Although what have been dealt with are the systems with first order differences, the key points are available for the systems with higher order differences. Obviously, both approaches are different from either Lee's discrete variation with variable time-steps [19], [20, [21] or Veselov's one with fixed time-steps for the discrete classical mechanics [28], [24]. They are also different from the discrete variation approach to field theory in [23] that is a generalization of Veselov's approach. In our approaches the differences with either variable step-lengths or the fixed ones are regarded as discrete derivatives being entire geometric objects. This is more obvious and natural from the viewpoint of noncommutative geometry and more analogical to the continuous mechanics and field theory. Therefore, in the continuous limit, the results given here by the variable difference variational approach unaffectedly lead to the correct continuous counterparts not only for the equations of motion and symplectic or multisymplectic preserving properties, but also for the conservation laws, especially for the energy conservation.

In view of the structure-preserving criterion for the discrete systems, there are more advantages for the variable difference discrete variational approach. Eventually, this has been already seen in [6] where in taking the continuous limits for the discrete variation problems, which is a generalized version of Lee-Veselov's variation, combining first discrete objects into some difference form is more controllable.

With variable step-lengths it is, of course, more or less straightforward to generalize the symplectic and multisyplectic schemes as ones that are not only symplectic and multisymplectic preserving but also discretely energy conserved as has been done for variational symplectic energy-momentum integrators in discrete Lagrangian formalism [18], [6], and in discrete Hamiltonian formalism [7]. But, these discrete formalisms do not transform to each other via discrete Legendre transformation.

The difference variational approach has been applied to the symplectic algorithm and multisymplectic one for both Lagrangian and Hamiltonian formalism in [14]. It has been shown that the discrete integrants can be combined together in certain manner as certain geometric objects in order to construct some numerical schemes with fixed step-lengths as variational integrators such as the midpoint scheme in symplectic algorithm and the Preissman scheme in multisymplectic algorithm. Obviously, the variable difference variational approach should be able to apply to the symplectic and multisymplectic algorithms with variable step-lengths for both Lagrangian and Hamiltonian formalism. This issue will be published in details elsewhere.

It has been found that the necessary and sufficient conditions for the symplectic 2-form preserving in mechanics and the multisymplectic 2 -forms preserving in field theories are the corresponding Euler-Lagrange 1-form is closed in the relevant Euler-Lagrange cohomology [12], [13. This is also true for the discrete cases [12], [14 as well as the symplectic and multisymplectic algorithms [14]. For the cases studied in this paper, the Euler-Lagrange cohomology should also be true for the various variation problems with variable domains or step-lengths. In fact, this matter is already indicated by the boundary terms in the vertical parts of these variation problems. We will explain this issue in more detail elsewhere. 
We have almost completely employed the ordinary description in a coordinate manner in this paper not only in order to be more easily understood, especially for non-mathematician, but for dealing with both continuous and discrete cases in an analogical manner. It should be mentioned however that both the variation problems and the Euler-Lagrange cohomology for continuous cases could be dealt with in a coordinate free version in terms of jet bundle and variational bicomplex (see, for example, [2], [3]). Although as far as the local issues are concerned, the essentials are almost the same. The coordinate free expression should be able to contain more general and complicated cases with nontrivial topology. On the other hand, however, for the discrete cases the ordinary jet bundle and variational bicomplex approach should be generalized to the ones include non-commutative differential calculus in principle. We will present the variational bicomplex approach to the issues in this paper elsewhere, specially the one with non-commutative differential calculus to the discrete cases [17].

\section{Acknowledgments}

We would like to thank Profs/Drs/Mr/Ms J.B. Chen, Y.Q. Li, T.T. Liu, M.Z. Qin, Z.J. Shang, G. Sun, Y.J. Sun, Y.F. Tan, Y.S. Wang, X.N. Wu and Z. Xu for valuable discussions. This work was supported in part by the National Natural Science Foundation of China and the National Key Project for Basic Research of China G1998030601. 


\section{REFERENCES}

[1] R. Abraham and J.E. Marsden, Foundation of Mechanics, (1978), (Second Ed.) AddisonWesley.

[2] I.M. Anderson, The variational bicomplex, (1989) appeared as a technical report on http ://www.math.usu.edu $f g \_m p$ in 2001.

[3] I.M. Anderson, "Introduction to the variational bicomplex", in Mathematical Aspects of Classical Field Theory, ed. M. Gotay, J. Marsden, V. Moncrief, Cont. Math. 132 (1992) 51-73.

[4] V.I. Arnold, Mathematical Methods of Classical Mechanics, Graduate texts in Math. 60 (1978), (Second Ed.) Springer-Verlag, (1989).

[5] T.J. Bridges, Multisymplectic Structures and Wave Propagation, Math. Proc. Camb. Phil. Soc. 121 (1997) 147-190.

[6] J.B. Chen, H.Y. Guo and K. Wu, Total variation and variational symplectic energymomentum integrators, Preprint arXiv:hep-th/0109178.

[7] J.B. Chen, H.Y. Guo and K. Wu, Total variation in Hamiltonian formalism and symplectic-energy integrators, Preprint arXiv:hep-th/0111185.

[8] R. Courant and D. Hilbert, Methods of Mathematical Physics, Interscience, New York, (1953).

[9] W. Dittrich and M. Reuter, "Classical and quantum dynamics", Springer-Verlag (1994).

[10] K. Feng, On difference schemes and symplectic geometry, Proc. of the 1984 Beijing Symposium on Differential Geometry and Differential Equations - Computation of Partial Differential Equations, Ed. by Feng Keng, Science Press, Beijing, 1985.

Selected Works of Feng Keng II (1995) and the references therein.

[11] Z. Ge and J.E. Marsden, Lie-Poisson integrators and Lie-Poisson Hamilton-Jacobi theory, Phys. Lett. A133, 134-139, (1988).

[12] H.Y. Guo, Y.Q. Li and K. Wu, On symplectic and multisymplectic structures and their discrete versions in Lagrangian formalism, Comm. Theor. Phys., 35, 703-710, (2001).

[13] H.Y. Guo, Y.Q. Li, K. Wu and S.K. Wang, Symplectic, multisymplectic structures and Euler-Lagrange cohomology, Preprint AS-ITP-2001-009, arXiv:hep-th/0104140.

[14] H.Y. Guo, Y.Q. Li, K. Wu and S.K. Wang, Difference discrete variational principle, Euler-Lagrange cohomology and symplectic, multisymplectic structures, Preprint arXiv:hep-th/0106001.

H.Y. Guo, Y.Q. Li, K. Wu and S.K. Wang, Difference discrete variational principle, Euler-Lagrange cohomology and symplectic, multisymplectic structures I: Difference discrete variational principle, Comm. Theor. Phys. (Beijing), 37 (2002) 1-10; II: EulerLagrange Cohomology, ibid, to appear; III: Application to Symplectic and Multisymplectic Algorithms, ibid, to appear.

[15] H.Y. Guo, K. Wu, S.H. Wang, S.K. Wang and G.M. Wei, Noncommutative Differential Calculus Approach to Symplectic Algorithm on Regular Lattice, Comm. Theor. Phys. 34 (2000) 307-318.

[16] H.Y. Guo, K. Wu and W. Zhang, Noncommutative Differential Calculus on Abelian Groups and Its Applications, Comm. Theor. Phys. 34 (2000) 245-250.

[17] H.Y. Guo, K. Wu et al. In preperation.

[18] C. Kane, J.E. Marsden and M. Ortiz, Symplectic-energy-momentum preserving variational integrators, J. Math. Phys., 40, 3353-3371 (1999).

[19] T.D. Lee, Can time be a discrete dynamical variable? Phys. Lett., 122B, 217-220 (1983). 
[20] T.D. Lee, Difference equations and conservation laws, J. Statis. Phys., 46, 843-860, (1987).

[21] T.D. Lee, Discrete mechanics, Lectures given at the International School of Subnuclear Physics, Erice, August 1983.

[22] J.E. Marsden and S. Shkoller, Multisymplectic Geometry, Covariant Hamiltonian and Water Waves, Math. Proc. Camb. Phil. Soc. 125 (1999) 535-575.

[23] J.E. Marsden, G.W. Patrick and S. Shkoller, Multisymplectic Geometry, Variational Integrators, and Nonlinear PDEs, Comm. Math. Phys. 199 (1998) 351-395.

[24] J. Moser and A.P. Veselov, Discrete Versions of Some Classical Integrable Systems and Factorization of Matrix Polynomials, Comm. Math. Phys. 139 (1991) 217-243.

[25] P.J. Olver, Applications of Lie Groups to Differential Eequations. Second Ed. GTM 107, Springer-Verlag (1993).

[26] R.D. Ruth, A Canonical Integration Technique. IEEE Trans. Nucl. Sci.. 30 (1983), 1669-2671.

[27] J.M. Sanz-Serna and M.P. Calvo, Numerical Hamiltonian Problems, Chapman and Hall, London. 1994 and references therein.

[28] A.P. Veselov, Integrable Discrete-time Systems and Difference Operators, Funkts. Anal. Prilozhen, 22 (1988) 1-13. 